TRANSACTIONS OF THE

AMERICAN MATHEMATICAL SOCIETY

Volume 350, Number 12, December 1998, Pages 4799-4838

S $0002-9947(98) 02083-2$

\title{
SYMMETRIC FUNCTIONAL DIFFERENTIAL EQUATIONS AND NEURAL NETWORKS WITH MEMORY
}

\author{
JIANHONG WU
}

\begin{abstract}
We establish an analytic local Hopf bifurcation theorem and a topological global Hopf bifurcation theorem to detect the existence and to describe the spatial-temporal pattern, the asymptotic form and the global continuation of bifurcations of periodic wave solutions for functional differential equations in the presence of symmetry. We apply these general results to obtain the coexistence of multiple large-amplitude wave solutions for the delayed Hopfield-Cohen-Grossberg model of neural networks with a symmetric circulant connection matrix.
\end{abstract}

\section{INTRODUCTION}

The purpose of this paper is to study the spatial-temporal patterns of solutions for systems of functional differential equations in the presence of symmetry. Of major concern is the existence, the asymptotic form, the isotropy group and the global continuation of periodic wave solutions. The well-known Hopfield-CohenGrossberg model of neural networks with delay provides the motivation and the illustration of our main general results.

We will start with the symmetric local Hopf bifurcation problem of the following parametrized system of functional differential equations

$$
\dot{x}(t)=L(\alpha) x_{t}+f\left(\alpha, x_{t}\right),
$$

where $f(\alpha, 0)=0$ and $\frac{\partial}{\partial \phi} f(\alpha, 0)=0$ for $\alpha \in \mathbb{R}$ and $\phi \in C:=C\left([-\tau, 0] ; \mathbb{R}^{n}\right)$, $\tau \geq 0$, is a given constant, $x_{t}$ is the usual notation for an element of $C$ defined by $x_{t}(s)=x(t+s)$ with $s \in[-\tau, 0], L: \mathbb{R} \times C \rightarrow \mathbb{R}^{n}$ is continuous and linear in the second argument. Moreover, there exists a compact Lie group $\Gamma$ acting on $\mathbb{R}^{n}$ such that $f(\alpha, \gamma \phi)=\gamma f(\alpha, \phi)$ and $L(\alpha) \gamma \phi=\gamma L(\alpha) \phi$ for $(\alpha, \gamma, \phi) \in \mathbb{R} \times \Gamma \times C$, where $\gamma \varphi \in C$ is given by $(\gamma \phi)(s)=\gamma \phi(s)$ for $s \in[-\tau, 0]$. We assume that there exists a critical value $\alpha_{0}$ such that at $\alpha=\alpha_{0}$, (i). The infinitesimal generator $A(\alpha)$ of the $C_{0}$-semigroup generated by the linear system $\dot{x}(t)=L(\alpha) x_{t}$ has a pair of purely imaginary eigenvalues $\pm i \beta_{0}$; (ii) the generalized eigenspace $U_{i \beta_{0}}\left(A\left(\alpha_{0}\right)\right)$ associated with $i \beta_{0}$ consists of eigenvectors of $A\left(\alpha_{0}\right)$ only and the restricted action of $\Gamma$ on $U_{i \beta_{0}}\left(A\left(\alpha_{0}\right)\right)$ is isomorphic to $V \oplus V$ for some absolutely irreducible representation $V$ of $\Gamma$.

Received by the editors September 13, 1995.

1991 Mathematics Subject Classification. Primary 34K15, 34K20, 34C25.

Key words and phrases. Periodic solution, delay differential equation, wave, symmetry, neural network, equivariant degree, global bifurcation.

Research partially supported by the Natural Sciences and Engineering Research Council of Canada.

(C)1998 American Mathematical Society 
Under the above assumptions, $U_{i \beta_{0}}\left(A\left(\alpha_{0}\right)\right)$ consists of $\omega=\frac{2 \pi}{\beta_{0}}$-periodic functions and $\Gamma \times S^{1}$ acts on $U_{i \beta_{0}}\left(A\left(\alpha_{0}\right)\right)$ by shifting arguments. We will show, in Theorem 2.1, that under usual non-resonance and transversality conditions, for every subgroup $\Sigma \leq \Gamma \times S^{1}$ such that the $\Sigma$-fixed point subspace of $U_{i \beta_{0}}\left(A\left(\alpha_{0}\right)\right)$ is of dimension 2, system (1.1) has a bifurcation of periodic solutions whose spatiotemporal symmetry can be completely characterized by $\Sigma$. In other words, under non-resonance and transversality conditions, maximal isotropy groups with minimal-dimensional fixed-point subspaces lead to bifurcations of periodic solutions with a certain spatio-temporal symmetry.

Note that the presence of symmetry often causes purely imaginary eigenvalues to be multiple and hence, the standard Hopf bifurcation theory of functional differential equations (cf. Hale [27], Hassard, Kazarinoff and Wan [29]) cannot be applied. Our general result, Theorem 2.1, represents an analog for functional differential equations of a well-known symmetric Hopf bifurcation theorem established by Golubitsky and Stewart [24] for ordinary differential equations (see also Golubitsky, Stewart and Schaeffer [25] and Vanderbauwhede [54] for a more detailed account of the local symmetric Hopf bifurcation theory). However, the proof of this analogue is not an elementary exercise as even the precise statement and verification of the hypotheses of this analog require nontrivial applications of some important facts of the generalized eigenspaces of the infinitesimal generators of solution semigroups and the decomposition theory of linear retarded functional differential equations.

We next consider the global continuation of the bifurcation of symmetric periodic solutions detected by the analytic local symmetric Hopf bifurcation theorem (Theorem 2.1). Our strategy here is strongly influenced by the work of Alexander and Auchmuty [3] where the global continuation of phase-locked oscillations in a system of ordinary differential equations arising from Turing rings of identical cells was investigated by considering the maximal continuation of periodic solutions for an associated scalar functional differential equations of mixed type (with both advanced and delayed arguments). More generally, our analytic local symmetric Hopf bifurcation theorem describes the spatio-temporal pattern of the bifurcated periodic solutions. This spatio-temporal pattern of the bifurcated periodic solutions often enables us to obtain a relatively simpler system of functional differential equation of mixed type and with an additional parameter (usually the period) which completely characterizes the bifurcated periodic solutions. In other words, the global continuation problem of the bifurcated symmetric periodic solutions of a parametrized system of functional differential equations can be reduced to a corresponding problem of the bifurcated periodic solutions of a relatively simpler system of functional differential equations which is of mixed type and with two parameters, but without symmetry.

With this reduction in mind, we apply the $S^{1}$-bifurcation theory developed by Geba and Marzantowicz [21] to establish a global Hopf bifurcation theorem, Theorem 3.3, for a system of functional differential equations of mixed type and with two parameters. At each stationary solution a sequence of bifurcation invariants, called the crossing numbers, is defined and can be evaluated from the information, via the Brouwer degree, of the linearization of the system at the stationary solution. Our global bifurcation theorem then claims that in the so-called Fuller's space (cf. Fuller [19]), along each bounded connected component of the nontrivial periodic solutions, the sum of the aforementioned crossing numbers must be zero. 
Global bifurcation problems have been extensively studied during the last two decades. A global bifurcation theorem was first established by Rabinowitz [51] for a compact perturbation of the identity parametrized by a real number, and then generalized to more general classes of nonlinear mappings by Alexander [1], Alexander and Fitzpatrick [4], Dancer [11], Fenske [14], Hetzer and Stallbohm [31], Nussbaum [48] and Stuart [53], to name a few. A similar result was also obtained for global continua of periodic solutions of differential equations by Alexander [1], Alexander and Fitzpatrick [4], Alexander and Yorke [5], Chow and Mallet-Paret [8], Chow, Mallet-Paret and Yorke [9], Fiedler [15, 16], Fitzpatrick, [17], Geba and Marzantowicz [21], Ize [35, 36], Ize, Massabó and Vignoli [37, 38], Mallet-Paret and Yorke [46], Nussbaum [49]. We refer to Fielder [15], Ize, Massabó and Vignoli $[37,38]$ and Erbe, Geba, Krawcewicz and $\mathrm{Wu}[20]$ for a detailed account of the literature.

Our general result, Theorem 3.3, represents an analog of the aforementioned results, in particular, the results of Alexander and Auchmuty [2], Chow and MalletParet [8], Fiedler [15, 16], Ize [35, 36], Mallet-Paret and Yorke [46] and Nussbaum [49], for functional differential equations of mixed type and with two parameters. This is not strikingly new but provides a crucial tool in our later application to the existence of large-amplitude wave solutions of delayed neural networks. It should be mentioned that in our approach, we employed the $S^{1}$-equivariant degree constructed by Dylawerski, Geba, Jodel and Marzantowicz [12]. There is now a more general version of the equivariant degree introduced by Ize, Massabó and Vignoli [37, 38] and Geba, Krawcewicz and $\mathrm{Wu}[20]$ and a corresponding bifurcation theory developed by Ize, Massabó and Vignoli [37, 38], Krawcewicz, Vivi and Wu [39] and Krawcewicz and $\mathrm{Wu}$ [40]. We emphasize that the main contribution of Theorem 3.3 is to formulate the global bifurcation theory in the setting easily applied to the problem of obtaining an unbounded continuation of periodic solutions of functional differential equations.

In Sections 4 and 5, our general results will be illustrated by their applications to the delayed Hopfield-Cohen-Grossberg model of neural networks:

$$
\dot{u}_{i}(t)=-u_{i}(t)+\sum_{j=1}^{n} J_{i j} f\left(u_{j}(t-\tau)\right), \quad 1 \leq i \leq n,
$$

where $f$ is a sigmoidal function normalized so that $f(0)=0, J=\left(J_{i j}\right)$ is a symmetric circulant matrix with all the diagonal elements identical to zero. It was shown by Hopfield [33, 34] and Cohen and Grossberg [10] that every solution of (1.2) is convergent to the set of equilibria if $\tau=0$. On the other hand, in electronic implementations of analog neural networks, time delays are present in the communication and response of neurons due to the finite switching speed of amplifiers (neurons). Designing a network to operate more quickly increases the relative size of the intrinsic delay and may cause sustained oscillations. In particular, Marcus and Westervelt [47] have demonstrated how the neuron gain, delay and the size and connection topology of the network affect the existence of oscillatory modes in continuous-time analog neural networks with time delay from the viewpoint of local analysis and with the help of numerical integration and some experiments on a small electronic network. 
By using Theorem 2.1 and Theorem 3.3, we will show that system (1.2) exhibits very rich dynamics and various types of oscillations for large delay. In particular, we will obtain the coexistence of

(i) pitchfork bifurcations of equilibria;

(ii) Hopf bifurcations of synchronous oscillations (periodic solutions satisfying $u_{i}(t)=u_{i-1}(t)$ for $\left.i(\bmod n), t \in \mathbb{R}\right)$;

(iii) Hopf bifurcations of phase-locked oscillations (periodic solutions satisfying $u_{i}(t)=u_{i-1}\left(t-\frac{r}{n} p\right), i(\bmod n), t \in \mathbb{R}$, where $r \in\{0, \ldots, n-1\}$ is a given integer and $p>0$ is a period of $u$ );

(iv) Hopf bifurcations of mirror-reflecting waves (periodic solutions satisfying $\left.u_{i}(t)=u_{n-i}(t), i(\bmod n), t \in \mathbb{R}\right)$;

(v) Hopf bifurcations of standing waves (periodic solutions satisfying $u_{n-i}(t)=$ $u_{i}\left(t-\frac{1}{2} p\right), i(\bmod n), t \in \mathbb{R}$, where $p>0$ is a period of $\left.u\right)$.

We will also show that several branches of phase-locked oscillations, standing waves and mirror-reflecting waves may bifurcate simultaneously from the trivial solution at some critical values of the delay.

The above wave solutions are special cases of the so-called coherent oscillation observed by Marcus and Westervelt [47]. In comparison with these results, we not only can detect the existence of sustained oscillations but also describe their spatio-temporal patterns. Moreover, as a result of our approach based on global bifurcation theorems, the coexistence of the aforementioned wave solutions will be established for delay not only near but also far away form the critical values. Therefore, we can obtain wave solutions of large amplitudes. We establish such global existence results by applying Theorem 3.3 and by (i) obtaining a priori bounds for periodic solution; (ii) excluding wave solutions of a certain period using the spectral analysis of Nussbaum [50] for circulant matrices (see Lemma 5.3, Theorem 5.4 and Theorem 6.3).

Depending on the value of the neuron gain $\left(f^{\prime}(0)\right)$ and the topology and size of the interconnection matrix, the aforementioned oscillations can be either stable or unstable. In fact, in Marcus and Westervelt [47] it has been observed that some systems of neural networks with delay possess multiple basins of attraction for coexisting equilibria and oscillatory attractors. Due to the (topological) nature of our approach to the existence of wave solutions we are, in general, unable to discuss the stability of these waves, except in several special cases where the theory of monotone dynamical systems of Hirsch [32] and Smith [52] can be applied to exclude stable discrete waves, as will be illustrated in Section 6 .

Finally, we mention that existing research has indicated some similarity between the system (1.2) with large delay and the parallel-update network $u_{i}(k+1)=$ $\sum_{j=1}^{n} J_{i j} f\left(u_{j}(k)\right)$ studied in Frumkin and Moses [18], Goles-Chacc, FogelmanSoulie and Pellegrin [22], Goles and Vichniac [23], Grinstein, Jayaprakash and He [26], Little [43] and Little and Shaw [44]. We wish to address the related singular perturbation problem in a future paper.

\section{Analytic local Symmetric Hopf Bifurcations for FDEs}

Let $\tau \geq 0$ be a given real number and $C$ denote the Banach space of continuous mapping from $[-\tau, 0]$ into $\mathbb{R}^{n}$ equipped with the supremum norm $\|\phi\|=$ $\sup _{-\tau \leq \theta \leq 0}|\phi(\theta)|$ for $\phi \in C$. In what follows, if $\sigma \in \mathbb{R}, A \geq 0$ and $x:[\sigma-\tau, \sigma+A] \rightarrow$ 
$\mathbb{R}^{n}$ is a continuous mapping, then $x_{t} \in C, t \in[\sigma, \sigma+A]$, is defined by $x_{t}(\theta)=x(t+\theta)$ for $-\tau \leq \theta \leq 0$.

Suppose $L: \mathbb{R} \times C \rightarrow \mathbb{R}^{n}$ is continuous and linear in the second argument, $f: \mathbb{R} \times C \rightarrow \mathbb{R}^{n}$ is continuous and has continuous first and second derivatives in the second argument with $f(\alpha, 0)=0, \frac{\alpha f}{\alpha \phi}(\alpha, 0)=0$ for $\alpha \in \mathbb{R}$ and $\phi \in C$. Consider the following system of delay differential equations

$$
\dot{x}(t)=L(\alpha) x_{t}+f\left(\alpha, x_{t}\right),
$$

where $\dot{x}(t)$ denotes $\frac{d}{d t} x(t)$. It is well-known that for each fixed $\alpha$, the linear system

$$
\dot{x}(t)=L(\alpha) x_{t}
$$

generates a strongly continuous semigroup of linear operators with the infinitesimal generator $A(\alpha)$ given by

$$
\begin{aligned}
A(\alpha) \phi & =\dot{\phi}, \quad \phi \in \operatorname{Dom}(A(\alpha)), \\
\operatorname{Dom}(A(\alpha)) & =\{\phi \in C ; \dot{\phi} \in C, \dot{\phi}(0)=L(\alpha) \phi\} .
\end{aligned}
$$

Moreover, the spectrum $\sigma(A(\alpha))$ of $A(\alpha)$ consists of eigenvalues which are solutions of the following characteristic equation

$$
\operatorname{det} \Delta(\alpha, \lambda)=0
$$

where the characteristic matrix $\Delta(\alpha, \lambda)$ is given by

$$
\Delta(\alpha, \lambda)=\lambda \operatorname{Id}-L(\alpha)\left(e^{\lambda \cdot} \operatorname{Id}\right) .
$$

We assume

(H1) The characteristic matrix is continuously differentiable in $\alpha \in \mathbb{R}$ and there exist $\alpha_{0} \in \mathbb{R}$ and $\beta_{0}>0$ such that (i) $A\left(\alpha_{0}\right)$ has eigenvalues $\pm i \beta_{0}$; (ii) the generalized eigenspace, denoted by $U_{i \beta_{0}}\left(A\left(\alpha_{0}\right)\right)$, of these eigenvalues $\pm i \beta_{0}$ consists of eigenvectors of $A\left(\alpha_{0}\right)$; (iii) all other eigenvalues of $A\left(\alpha_{0}\right)$ are not integer multiple of $\pm i \beta_{0}$.

(H2) There exists a compact Lie group $\Gamma$ acting on $\mathbb{R}^{n}$ such that both $L(\alpha)$ and $f(\alpha, \cdot)$ are $\Gamma$-equivariant, i.e. $f(\alpha, \gamma \phi)=\gamma f(\alpha, \phi), L(\alpha) \gamma \phi=\gamma L(\alpha) \phi$ for $(\alpha, \gamma, \phi) \in \mathbb{R} \times \Gamma \times C$, where $\gamma \phi \in C$ is given by $(\gamma \phi)(\theta)=\gamma \phi(\theta)$, $\theta \in[-\tau, 0]$.

Note that we do not require the eigenvalues $\pm i \beta_{0}$ to be simple. In fact, the presence of symmetry often causes these purely imaginary eigenvalues to be multiple. Hence, the standard Hopf bifurcation theory of functional differential equations (cf. Hale [27] and Hassard, Kazarinoff and Wan [29]) cannot be applied. To state the next assumption, we note that under $(\mathrm{H} 1), U_{\beta_{0}}\left(A\left(\alpha_{0}\right)\right)$ is the real vector space consisting of $\operatorname{Re}\left(e^{i \beta_{0} \cdot b}\right)$ and $\operatorname{Im}\left(e^{i \beta_{0} \cdot b}\right)$ such that $b \in \operatorname{Ker} \Delta\left(\alpha_{0}, i \beta_{0}\right)$. Moreover, there exists a natural identification between $\operatorname{Ker} \Delta\left(\alpha_{0}, i \beta_{0}\right)$ and $\mathbb{R}^{2 m}$, where $2 m=\operatorname{dim} \operatorname{Ker} \Delta\left(\alpha_{0}, i \beta_{0}\right)$, as a real vector space. We also require

(H3) There exists an $m$-dimensional absolutely irreducible representation $V$ of $\Gamma$ such that $\operatorname{Ker} \Delta\left(\alpha_{0}, i \beta_{0}\right)$ is isomorphic to $V \oplus V$, here a representation $V$ of $\Gamma$ is absolutely irreducible if the only linear mapping that commutes with the action of $\Gamma$ is a scalar multiple of the identity.

Remark 2.1. Assume that (H1)-(H3) are satisfied. Let $\left\{b_{j 1}+i b_{j 2}\right\}_{j=1}^{m}$ be a basis for Ker $\Delta\left(\alpha_{0}, i \beta_{0}\right)$ and define $\sin _{\beta}, \cos _{\beta} \in C([-\tau, 0] ; \mathbb{R})$ by

$$
\sin _{\beta}(\theta)=\sin (\beta \theta), \quad \cos _{\beta}(\theta)=\cos (\beta \theta), \quad \theta \in[-\tau, 0] .
$$


Then the columns of $\Phi_{\alpha_{0}}=\left(\varepsilon_{1}, \ldots, \varepsilon_{2 m}\right)$ form a basis for $U_{i \beta_{0}}\left(A\left(\alpha_{0}\right)\right)$, where

$$
\begin{aligned}
\varepsilon_{j} & =\sin _{\beta} b_{j 1}+\cos _{\beta} b_{j 2}, \\
\varepsilon_{m+j} & =\cos _{\beta} b_{j 1}-\sin _{\beta} b_{j 2}, \quad 1 \leq j \leq m .
\end{aligned}
$$

It can be easily verified that

$$
A\left(\alpha_{0}\right) \varepsilon_{j}=\beta \varepsilon_{m+j}, \quad A\left(\alpha_{0}\right) \varepsilon_{m+j}=-\beta \varepsilon_{j}, \quad 1 \leq j \leq m .
$$

That is,

$$
A \Phi_{\alpha_{0}}=\Phi_{\alpha_{0}} B\left(\alpha_{0}\right)
$$

where

$$
B\left(\alpha_{0}\right)=\left(\begin{array}{cc}
0 & \beta \operatorname{Id}_{m} \\
-\beta \operatorname{Id}_{m} & 0
\end{array}\right)
$$

and $\operatorname{Id}_{m}$ is the identity matrix of order $m$.

Remark 2.2. Let

$$
L(\alpha) \phi=\int_{-\tau}^{0}[d \eta(\alpha, \theta)] \phi(\theta), \quad \phi \in C, \alpha \in \mathbb{R},
$$

where $\eta(\alpha, \theta)$ is an $n \times n$ matrix function of bounded variation in $\theta \in[-\tau, 0]$. Then (2.2) can be written as

$$
\dot{x}(t)=\int_{-\tau}^{0}[d \eta(\alpha, \theta)] x(t+\theta) .
$$

Along with this linear homogeneous equation, we will also consider the formal adjoint equation

$$
\dot{y}(s)=-\int_{-\tau}^{0} y(s-\theta)[d \eta(\alpha, \theta)]
$$

together with the bilinear form

$$
(\psi, \phi)_{\alpha}=\psi(0) \phi(0)-\int_{-\tau}^{0} \int_{0}^{\theta} \psi(\xi-\theta)[d \eta(\alpha, \theta)] \phi(\xi) d \xi
$$

for $\phi \in C$ and $\psi \in C^{*}:=C\left([0, \tau] ; \mathbb{R}^{n *}\right)$, where $\mathbb{R}^{n *}$ is the space of $n$-dimensional row vectors. Let $A^{*}(\alpha)$ denote the infinitesimal generator of the strongly continuous semigroup generated by (2.6) on $C^{*}$. Then the subspace of solutions to the linear algebraic equation

$$
x^{*} \Delta\left(\alpha_{0}, i \beta_{0}\right)=0, \quad x^{*} \in \mathbb{R}^{n *}+i \mathbb{R}^{n *}:=\mathbb{C}^{n *}
$$

has a basis $\left\{c_{j 1}+i c_{j 2}\right\}_{j=1}^{m}, c_{j 1}, c_{j 2} \in \mathbb{R}^{n *}, 1 \leq j \leq m$. If $\cos _{\beta}^{*}, \sin _{\beta}^{*} \in C([0, \tau] ; \mathbb{R})$ are defined by

$$
\cos _{\beta}^{*}(\theta)=\cos (\beta \theta), \sin _{\beta}^{*}(\theta)=\sin (\beta \theta), \quad \theta \in[0, \tau],
$$

then $\Psi_{\alpha_{0}}^{*}=\left(\varepsilon_{1}^{*}, \ldots, \varepsilon_{2 m}^{*}\right)$ forms a basis for $U_{i \beta_{0}}\left(A^{*}\left(\alpha_{0}\right)\right)$, where

$$
\begin{aligned}
& \varepsilon_{j}^{*}=\sin _{\beta}^{*} c_{j 1}+\cos _{\beta}^{*} c_{j 2}, \\
& \varepsilon_{m+j}^{*}=\cos _{\beta}^{*} c_{j 1}-\sin _{\beta}^{*} c_{j 2}, \quad 1 \leq j \leq m .
\end{aligned}
$$


Lemma 2.1. Under assumptions $(\mathrm{H} 1)-(\mathrm{H} 3)$, there exist $\delta_{0}>0$ and a continuously differentiable function $\lambda:\left(\alpha_{0}-\delta_{0}, \alpha_{0}+\delta_{0}\right) \rightarrow \mathbb{C}$ such that $\lambda\left(\alpha_{0}\right)=i \beta_{0}$, $\lambda(\alpha)$ is an eigenvalue of $A(\alpha), U_{\lambda(\alpha)}(A(\alpha))$ consists of eigenvectors of $A(\alpha)$, and $\operatorname{dim} U_{\lambda(\alpha)}(A(\alpha))=\operatorname{dim} U_{i \beta_{0}}\left(A\left(\alpha_{0}\right)\right)$. Moreover, there exist $2 m$ continuously differentiable mappings $e_{j}:\left(\alpha_{0}-\delta_{0}, \alpha_{0}+\delta_{0}\right) \rightarrow \mathbb{C}$ and $2 m$ continuously differentiable mappings $e_{j}^{*}:\left(\alpha_{0}-\delta_{0}, \alpha_{0}+\delta_{0}\right) \rightarrow C^{*}$ such that $e_{j}\left(\alpha_{0}\right)=\varepsilon_{j}, e_{j}^{*}\left(\alpha_{0}\right)=\varepsilon_{j}^{*}$, $\Phi_{\alpha}=\left(e_{1}(\alpha), \ldots, e_{2 m}(\alpha)\right)$ is a basis of $U_{\lambda(\alpha)}(A(\alpha))$ and $\Psi_{\alpha}=\left(e_{1}^{*}(\alpha), \ldots, e_{2 m}^{*}(\alpha)\right)$ is a basis of $U_{\lambda(\alpha)}\left(A^{*}(\alpha)\right)$.

Proof. Let $P$ and $I-P$ denote the projection operators defined by the decomposition

$$
\mathbb{C}^{n}=\operatorname{Ker} \Delta\left(\alpha_{0}, i \beta_{0}\right) \oplus \operatorname{Ran} \Delta\left(\alpha_{0}, i \beta_{0}\right) .
$$

As $\operatorname{Ker} \Delta\left(\alpha_{0}, i \beta_{0}\right)$ is $\Gamma$-invariant, $P$ and $I-P$ commutes with the $\Gamma$-action. Rewrite the equation

$$
\Delta(\alpha, \lambda) b=0
$$

as

$$
\left\{\begin{array}{l}
\Delta\left(\alpha_{0}, i \beta_{0}\right) d=[I-P]\left[\Delta\left(\alpha_{0}, i \beta_{0}\right)-\Delta(\alpha, \lambda)\right]\left(b_{0}+d\right) \\
P\left[\Delta\left(\alpha_{0}, i \beta_{0}\right)-\Delta(\alpha, \lambda)\right]\left(b_{0}+d\right)=0
\end{array}\right.
$$

where

$$
b=b_{0}+d
$$

is the unique decomposition such that

$$
b_{0} \in \operatorname{Ker} \Delta\left(\alpha_{0}, i \beta_{0}\right), \quad d \in \operatorname{Ran} \Delta\left(\alpha_{0}, i \beta_{0}\right) .
$$

Applying the implicit function theorem, we obtain $\delta_{1}>0$ and an $n \times n$ matrix $D^{*}(\alpha, \lambda)$, continuously differentiable for $\left|\alpha-\alpha_{0}\right|<\delta_{1}$ and $\left|\lambda-i \beta_{0}\right|<\sigma_{1}$, such that $D^{*}\left(\alpha_{0}, i \beta_{0}\right)=0, d=D^{*}(\alpha, \lambda) b_{0}$ is a solution of the first equation of (2.9), and $D^{*}(\alpha, \lambda)$ commutes with the action of $\Gamma$ on $\operatorname{Ker} \Delta\left(\alpha_{0}, i \beta_{0}\right)$. Therefore, the existence of an eigenvalue $\lambda$ near $i \beta_{0}$ for $\alpha$ near $\alpha_{0}$ is equivalent to the existence of a solution of

$$
f(\alpha, \lambda) b_{0}=0
$$

where

$$
f(\alpha, \lambda) b_{0}=P\left[\Delta\left(\alpha_{0}, i \beta_{0}\right)-\Delta(\alpha, \lambda)\right]\left[\operatorname{Id}_{m}+D^{*}(\alpha, \lambda)\right] b_{0} .
$$

Properly choosing a basis for $\mathbb{C}^{n}$, we may assume

$$
P=\left(\begin{array}{cc}
\operatorname{Id}_{m} & 0 \\
0 & 0
\end{array}\right), \Delta(\alpha, \lambda)=\left(\begin{array}{cc}
\Delta_{11}(\alpha, \lambda) & \Delta_{12}(\alpha, \lambda) \\
\Delta_{21}(\alpha, \lambda) & \Delta_{22}(\alpha, \lambda)
\end{array}\right)
$$

where $\Delta_{11}(\alpha, \lambda)$ is an $m \times m$ matrix, $\Delta_{22}(\alpha, \lambda)$ is of order $(n-m) \times(n-m)$ and

$$
\Delta\left(\alpha_{0}, i \beta_{0}\right)=\left(\begin{array}{cc}
0 & 0 \\
0 & \Delta_{22}\left(\alpha_{0}, i \beta_{0}\right)
\end{array}\right), \operatorname{det} \Delta_{22}\left(\alpha_{0}, i \beta_{0}\right) \neq 0 .
$$

Therefore,

$$
f(\alpha, \lambda)=-\left[\Delta_{11}(\alpha, \lambda)+\Delta_{12}(\alpha, \lambda) D^{*}(\alpha, \lambda)\right]
$$

Moreover, substituting $d$ by $D^{*}(\alpha, \lambda) b_{0}$ in the first equation of (2.9), we get

$$
\Delta_{21}(\alpha, \lambda)=-\Delta_{22}(\alpha, \lambda) D^{*}(\alpha, \lambda) \text {. }
$$


This implies that

$$
\begin{aligned}
\Delta(\alpha, \lambda) & =\left(\begin{array}{cc}
\Delta_{11}(\alpha, \lambda) & \Delta_{12}(\alpha, \lambda) \\
-\Delta_{22}(\alpha, \lambda) D^{*}(\alpha, \lambda) & \Delta_{22}(\alpha, \lambda)
\end{array}\right) \\
& =\left(\begin{array}{cc}
\operatorname{Id}_{m} & 0 \\
0 & \Delta_{22}(\alpha, \lambda)
\end{array}\right)\left(\begin{array}{cc}
\Delta_{11}(\alpha, \lambda) & \Delta_{12}(\alpha, \lambda) \\
-D^{*}(\alpha, \lambda) & \operatorname{Id}_{m}
\end{array}\right) .
\end{aligned}
$$

Consequently, from (2.12) it follows that

$$
\operatorname{det} \Delta(\alpha, \lambda)=(-1)^{m} \operatorname{det} \Delta_{22}(\alpha, \lambda) \operatorname{det} f(\alpha, \lambda) \text {. }
$$

As $\operatorname{dim} U_{i \beta_{0}}\left(A\left(\alpha_{0}\right)\right)=2 m$, by the well-known folk theorem for retarded functional differential equations (cf. Hale [27] and Levinger [42]) we have

$$
\left\{\begin{array}{l}
\left.\frac{\partial^{k}}{\partial \lambda^{k}} \operatorname{det} \Delta\left(\alpha_{0}, \lambda\right)\right|_{\lambda=i \beta_{0}}=0,0 \leq k \leq m-1, \\
\left.\frac{\partial^{m}}{\partial \lambda^{m}} \operatorname{det} \Delta\left(\alpha_{0}, \lambda\right)\right|_{\lambda=i \beta_{0}} \neq 0 .
\end{array}\right.
$$

Therefore, as $\operatorname{det} \Delta_{22}\left(\alpha_{0}, i \beta_{0}\right) \neq 0$, we derive from (2.14) the following

$$
\left\{\begin{array}{l}
\frac{\partial^{k}}{\partial \lambda^{k}} \operatorname{det} f(\alpha, \lambda)=0,0 \leq k \leq m-1, \\
\frac{\partial^{m}}{\partial \lambda^{m}} \operatorname{det} f(\alpha, \lambda) \neq 0 .
\end{array}\right.
$$

On the other hand, under assumption (H3) we may assume

$$
f(\alpha, \lambda)=\left(\begin{array}{ll}
F_{11}(\alpha, \lambda) & F_{12}(\alpha, \lambda) \\
F_{21}(\alpha, \lambda) & F_{22}(\alpha, \lambda)
\end{array}\right)
$$

for some $m \times m$ real matrices $F_{i j}, i, j=1,2$. The $\Gamma$-equivariance of $D^{*}(\alpha, \lambda)$ implies that $f(\alpha, \lambda)$ commutes with the diagonal action of $\Gamma$ on $V \oplus V$, and hence $F_{i j}$ commutes with the action of $\Gamma$ on $V$. By the absolute irreducibility of $V$, we have

$$
F_{i j}(\alpha, \lambda)=f_{i j}(\alpha, \lambda) \operatorname{Id}_{m}
$$

for some scalar functions $f_{i j}(\alpha, \lambda)$. So

$$
\operatorname{det} f(\alpha, \lambda)=q^{m}(\alpha, \lambda) \text {, }
$$

where

$$
q(\alpha, \lambda)=f_{11}(\alpha, \lambda) f_{22}(\alpha, \lambda)-f_{12}(\alpha, \lambda) f_{21}(\alpha, \lambda) .
$$

By (2.16), we get $q\left(\alpha_{0}, i \beta_{0}\right)=0,\left.\frac{\partial}{\partial \lambda} q\left(\alpha_{0}, \lambda\right)\right|_{\lambda=i \beta_{0}} \neq 0$. Therefore, from the implicit function theorem it follows that there exist $\delta_{0}>0$ and a continuous differentiable function $\lambda(\alpha)$ for $\left|\alpha-\alpha_{0}\right|<\delta_{0}$ such that $\lambda\left(\alpha_{0}\right)=i \beta_{0}, q(\alpha, \lambda(\alpha))=0$ and $\left|\lambda(\alpha)-i \beta_{0}\right|<\delta_{1}$. So, $\lambda(\alpha)$ is an eigenvalue of $A(\alpha)$ with multiplicity $2 m$, and the corresponding eigenvector is

$$
b(\alpha)=\left[I+D^{*}(\alpha, \lambda(\alpha))\right] b, \quad b \in \operatorname{Ker} \Delta\left(\alpha_{0}, i \beta_{0}\right) .
$$

Consequently, $\operatorname{dim} U_{\lambda(\alpha)}(A(\alpha))=\operatorname{dim} U_{i \beta_{0}}\left(A\left(\alpha_{0}\right)\right)$ for $\left|\alpha-\alpha_{0}\right|<\delta_{0}$. Let

$$
b_{j 1}^{*}(\alpha)+i b_{j 2}^{*}(\alpha)=\left[I+D^{*}(\alpha, \lambda(\alpha))\right]\left[b_{j 1}+i b_{j 2}\right] .
$$

Then

$$
\begin{gathered}
e_{j}(\alpha)=\operatorname{Im} e^{\lambda(\alpha) \cdot}\left[b_{j 1}^{*}(\alpha)+i b_{j 2}^{*}(\alpha)\right], \\
e_{m+j}(\alpha)=\operatorname{Re} e^{\lambda(\alpha) \cdot}\left[b_{j 1}^{*}(\alpha)+i b_{j 2}^{*}(\alpha)\right], \quad 1 \leq j \leq m,
\end{gathered}
$$

form a basis of $U_{\lambda(\alpha)}(A(\alpha))$ with the required properties. 
The same argument can be applied to construct the basis $\left\{c_{j 1}^{*}(\alpha)+i c_{j 2}^{*}(\alpha)\right\}_{j=1}^{m}$ for the subspace of solutions to the equation $x^{*} \Delta(\alpha, \lambda(\alpha))=0$ for $x^{*} \in R^{n *}$. Therefore,

$$
\begin{gathered}
e_{j}^{*}(\alpha)=\operatorname{Im} e^{\lambda(\alpha) \cdot}\left[c_{j 1}^{*}(\alpha)+i c_{j 2}^{*}(\alpha)\right], \\
e_{m+j}^{*}(\alpha)=\operatorname{Re} e^{\lambda(\alpha) \cdot}\left[c_{j 1}^{*}(\alpha)+i c_{j 2}^{*}(\alpha)\right], \quad 1 \leq j \leq m,
\end{gathered}
$$

form a basis of $U_{\lambda(\alpha)}\left(A^{*}(\alpha)\right)$ with the required properties. This completes the proof.

Remark 2.3. It can be easily shown that

$$
A(\alpha) \Phi_{\lambda(\alpha)}=\Phi_{\lambda(\alpha)} B(\alpha),
$$

where

$$
B(\alpha)=\left(\begin{array}{cc}
\operatorname{Re} \lambda(\alpha) \operatorname{Id}_{m} & -\operatorname{Im} \lambda(\alpha) \operatorname{Id}_{m} \\
\operatorname{Im} \lambda(\alpha) \operatorname{Id}_{m} & \operatorname{Re} \lambda(\alpha) \operatorname{Id}_{m}
\end{array}\right)
$$

We also need the following

Lemma 2.2. Let $\left(\Psi_{\alpha}, \Psi_{\alpha}\right)_{\alpha}=\left(\left(e_{j}^{*}, e_{k}\right)_{\alpha}\right)_{1 \leq j, k \leq 2 m}$. Then

$$
B^{\prime}(\alpha)=-\left(\Psi_{\alpha}, \Phi_{\alpha}\right)_{\alpha}^{-1} \Psi_{\alpha}(0)\left[\frac{d}{d \alpha} L(\alpha)\right] \Phi_{\alpha}
$$

The proof is similar to that of Lemma 3.9 on pp. 179 of Hale [27] and therefore is omitted.

Let $\omega=\frac{2 \pi}{\beta_{0}}$. Denote by $P_{\omega}$ the Banach space of all continuous $\omega$-periodic mappings $x: \mathbb{R} \rightarrow \mathbb{R}^{n}$. Then $\Gamma \times S^{1}$ acts on $P_{\omega}$ by

$$
(\gamma, \theta) x(t)=\gamma x(t+\theta), \quad(\gamma, \theta) \in \Gamma \times S^{1}, \quad x \in P_{\omega}
$$

Denote by $S P_{\omega}$ the subspace of $P_{\omega}$ consisting of all $\omega$-periodic solutions of (2.2) with $\alpha=\alpha_{0}$. Then for each subgroup $\Sigma \leq \Gamma \times S^{1}$, the fixed point set

$$
\operatorname{Fix}\left(\Sigma, S P_{\omega}\right)=\left\{x \in S P_{\omega} ;(\gamma, \theta) x=x \text { for all }(\gamma, \theta) \in \Sigma\right\}
$$

is a subspace.

Under assumption (H1), the columns of $U(t)=\Phi_{\alpha_{0}}(0) e^{B\left(\alpha_{0}\right) t}, t \in R$, form a basis for $S P_{\omega}$.

Lemma 2.3. $S P_{\omega}$ is a $\Gamma \times S^{1}$-invariant subspace of $P_{\omega}$.

Proof. For each $\gamma \in \Gamma, \gamma \operatorname{Ker} \Delta\left(\alpha_{0}, i \beta_{0}\right) \subseteq \operatorname{Ker} \Delta\left(\alpha_{0}, i \beta_{0}\right)$. So there exist $\alpha_{j k}^{\gamma}$ and $\beta_{j k}^{\gamma}, 1 \leq j, k \leq m$, such that

$$
\begin{aligned}
\gamma b_{j 1} & =\sum_{k=1}^{m}\left[\alpha_{j k}^{\gamma} b_{k 1}-\beta_{j k}^{\gamma} b_{k 2}\right], \\
\gamma b_{j 2} & =\sum_{k=1}^{m}\left[\beta_{j k}^{\gamma} b_{k 1}+\alpha_{j k}^{\gamma} b_{k 2}\right], \quad 1 \leq j \leq m .
\end{aligned}
$$

Let

$$
T_{1 \gamma}=\left(\alpha_{j k}^{\gamma}\right)_{1 \leq j, k \leq m}, \quad T_{2 \gamma}=\left(\beta_{j k}^{\gamma}\right)_{1 \leq j, k \leq m} .
$$

Then, we can easily verify that

$$
\gamma U(t)=U(t)\left(\begin{array}{cc}
T_{1 \gamma} & -T_{2 \gamma} \\
T_{2 \gamma} & T_{1 \gamma}
\end{array}\right)
$$


So $\gamma S P_{\omega} \subseteq S P_{\omega}$. Moreover, for every $\theta \in S^{1}$,

$$
\theta U(t)=U(t)\left(\begin{array}{cc}
\cos \theta \operatorname{Id}_{m} & -\sin \theta \operatorname{Id}_{m} \\
\sin \theta \operatorname{Id}_{m} & \cos \theta \operatorname{Id}_{m}
\end{array}\right) .
$$

Therefore, $\theta S P_{\omega} \subseteq S P_{\omega}$. This completes the proof.

Lemma 2.4. Let $T_{\gamma}=\left(\begin{array}{cc}T_{1 \gamma} & -T_{2 \gamma} \\ T_{2 \gamma} & T_{1 \gamma}\end{array}\right)$ be given in the argument of Lemma 2.3. Then $\Gamma \times S^{1}$ acts on $\mathbb{R}^{2 m}$ by

$$
(\gamma, \theta) w=T_{\gamma}\left(\begin{array}{cc}
\cos (\beta \theta) \operatorname{Id}_{m} & -\sin (\beta \theta) \operatorname{Id}_{m} \\
\sin (\beta \theta) \operatorname{Id}_{m} & \cos (\beta \theta) \operatorname{Id}_{m}
\end{array}\right) w, \quad w \in \mathbb{R}^{2 m},
$$

and this action is isomorphic to the restricted action of $\Gamma \times S^{1}$ on $U_{i \beta_{0}}\left(A\left(\alpha_{0}\right)\right)$.

Proof. Let $w=\left(w_{1}, \ldots, w_{2 m}\right)^{T} \in \mathbb{R}^{2 m}$. Then the mapping $H: \mathbb{R}^{2 m} \rightarrow U_{i \beta_{0}}\left(A\left(\alpha_{0}\right)\right)$ defined by

$$
H w=\sum_{j=1}^{2 m} w_{j} \varepsilon_{j}
$$

gives an isomorphism of the representation of $\mathbb{R}^{2 m}$ and $U_{i \beta_{0}}\left(A\left(\alpha_{0}\right)\right)$.

Lemma 2.5. Consider the linear nonhomogeneous equation

$$
\dot{x}(t)=L\left(\alpha_{0}\right) x_{t}+g(t) .
$$

Let

$$
T P_{\omega}=\left\{g \in P_{\omega} ;(2.17) \text { has an } \omega \text {-periodic solution }\right\} .
$$

Then $T P_{\omega}$ is $\Gamma \times S^{1}$-invariant.

Proof. It is well-known that (2.17) has an $\omega$-periodic solution if and only if

$$
\int_{0}^{\omega} y(t) g(t) d t=0
$$

for every $\omega$-periodic solution of the formal adjoint equation (2.6) with $\alpha=\alpha_{0}$. On the other hand, if $y(s)$ is a $\omega$-periodic solution of (2.6) with $\alpha=\alpha_{0}$, then

$$
\begin{aligned}
\dot{y}(s) \gamma & =-\int_{-\tau}^{0} y(s-\theta)\left[d \eta\left(\alpha_{0}, \theta\right)\right] \gamma \\
& =-\int_{-\tau}^{0} y(s-\theta) \gamma\left[d \eta\left(\alpha_{0}, \theta\right)\right] .
\end{aligned}
$$

So $y(s-\theta) \gamma$ is also an $\omega$-periodic solution of (2.6) with $\alpha=\alpha_{0}$. Consequently, for every $g \in T P_{\omega}$,

$$
\int_{0}^{\omega} y(t)(\gamma, \theta) g(t) d t=\int_{0}^{\omega} y(t) \gamma g(t+\theta) d \theta=\int_{0}^{\omega} y(t-\theta) \gamma g(t) d t=0 .
$$

This shows $(\gamma, \theta) g \in T P_{\omega}$, completing the proof.

Recall that in Lemma 2.1, we have proved that under $(\mathrm{H} 1)-(\mathrm{H} 3)$, there exist $\delta_{0}>0$ and a continuously differentiable function $\lambda:\left(\alpha_{0}-\delta_{0}, \alpha_{0}+\delta_{0}\right) \rightarrow \mathbb{C}$ such that $\lambda\left(\alpha_{0}\right)=i \beta_{0}$, and for each $\alpha \in\left(\alpha_{0}-\delta_{0}, \alpha_{0}+\delta_{0}\right), \lambda(\alpha)$ is an eigenvalue of $A(\alpha), U_{\lambda(\alpha)}(A(\alpha))$ consists of eigenvectors of $A(\alpha)$ and has the same dimension as $U_{i \beta_{0}}\left(A\left(\alpha_{0}\right)\right)$. We can now state the final assumption - the transversality condition:

(H4) $\left.\frac{d}{d \alpha} \operatorname{Re} \lambda(\alpha)\right|_{\alpha=\alpha_{0}} \neq 0$. 
Theorem 2.1. Assume that (H1)-(H4) are satisfied and $\operatorname{dim} \operatorname{Fix}\left(\Sigma, S P_{\omega}\right)=2$ for some $\Sigma \leq \Gamma \times S^{1}$. Then for a chosen basis $\left\{\delta_{1}, \delta_{2}\right\}$ of $\operatorname{Fix}\left(\Sigma, S P_{\omega}\right)$ there exist constants $a_{0}>0, a_{0}^{*}>0, \sigma_{0}>0$, functions $\alpha: \mathbb{R}^{2} \rightarrow \mathbb{R}, \omega^{*}: \mathbb{R}^{2} \rightarrow(0, \infty)$ and a continuous function $x^{*}: \mathbb{R}^{2} \rightarrow \mathbb{R}^{n}$, with all functions being continuously differentiable in $a \in \mathbb{R}^{2}$ with $|a|<a_{0}$, such that $x^{*}(a)$ is an $\omega(a)$-periodic solution of (2.1) with $\alpha=\alpha(a)$, and

$$
\begin{aligned}
\gamma x^{*}(a)(t) & =x^{*}(a)\left(t-\frac{\omega^{*}(a)}{\omega} \theta\right), \quad(\gamma, \theta) \in \Sigma, \\
x^{*}(0) & =0, \quad \omega^{*}(0)=\omega, \quad \alpha(0)=\alpha_{0}, \\
x^{*}(a) & =\left(\delta_{1}, \delta_{2}\right) a+o(|a|) \quad \text { as }|a| \rightarrow 0 .
\end{aligned}
$$

Furthermore, for $\left|\alpha-\alpha_{0}\right|<\alpha_{0}^{*}$, $\left|\omega^{*}-\frac{2 \pi}{\beta_{0}}\right|<\sigma_{0}$, every $\omega^{*}$-periodic solution of (2.1) with $\left\|x_{t}\right\|<\sigma_{0}, \gamma x(t)=x\left(t-\frac{\omega^{*}}{\omega} \theta\right)$ for $(\gamma, \theta) \in \Sigma, t \in \mathbb{R}$, must be of the above type.

Proof. We start with the normalization of the period. Let $\beta \in(-1,1), u(t)=$ $x((1+\beta) t)$. Then equation $(2.1)$ can be rewritten as

$$
\dot{u}(t)=L\left(\alpha_{0}\right) u_{t}+N\left(\alpha, \beta, u_{t}, u_{t, \beta}\right),
$$

where

$$
\begin{aligned}
u_{t, \beta}(\theta) & =u\left(t+\frac{\theta}{1+\beta}\right), \quad \theta \in[-\tau, 0], \\
N\left(\alpha, \beta, u_{t}, u_{t, \beta}\right) & =(1+\beta)\left[f\left(\alpha, u_{t, \beta}\right)+L(\alpha) u_{t, \beta}\right]-L\left(\alpha_{0}\right) u_{t} .
\end{aligned}
$$

Let $J: P_{\omega} \rightarrow T P_{\omega}$ be a fixed $\Gamma \times S^{1}$-equivariant projection and let $K: T P_{\omega} \rightarrow P_{\omega}$ be a bounded linear $\Gamma \times S^{1}$-equivariant operator such that $K g$ is an $\omega$-periodic solution of (2.17) for every $g \in T P_{\omega}$. Then $u$ is an $\omega$-periodic solution of (2.18) with $\Sigma$ as the group of symmetry if and only if there exists $z=\left(z_{1}, z_{2}\right)^{T} \in \mathbb{R}^{2}$ such that

$$
\left\{\begin{array}{l}
u=\left(\delta_{1}, \delta_{2}\right) z+K J N(\alpha, \beta, u ., u \cdot, \beta), \\
(I-J) N(\alpha, \beta, u ., u \cdot, \beta)=0 .
\end{array}\right.
$$

We now apply the implicit function theorem to obtain a solution of the first equation of (2.19), $u=u^{*}(\alpha, \beta, z)$, for $\alpha, \beta, z$ in a sufficiently small neighbourhood of $\left(\alpha_{0}, \beta_{0}, 0\right)$ such that

$$
u^{*}\left(\alpha_{0}, \beta_{0}, z\right)-\left(\delta_{1}, \delta_{2}\right) z=o(|z|) \quad \text { as }|z| \rightarrow 0 .
$$

The function $u^{*}(\alpha, \beta, z)$ is continuously differentiable in $(\alpha, z)$ from the implicit function theorem. Moreover, a solution of the first equation of (2.19) satisfies a differential integral equation and hence, $u^{*}(\alpha, \beta, z)(t)$ is differentiable in $t$. This, in turn, implies that $u^{*}(\alpha, \beta, z)$ is differentiable in $\beta$. Furthermore, the uniqueness guaranteed by the implicit function theorem and the $\Gamma$-equivariance of $f$ and $L$ imply that

$$
(\gamma, \theta) u^{*}(\alpha, \beta, z)=u^{*}(\alpha, \beta, z), \quad(\gamma, \theta) \in \Sigma .
$$

Consequently, all $\omega$-periodic solutions of (2.18) are obtained by finding solutions $(\alpha, \beta, z)$ of the bifurcation equation

$$
J N\left(\alpha, \beta, u_{.}^{*}(\alpha, \beta, z), u_{,, \beta}^{*}(\alpha, \beta, z)\right)=0 .
$$


As noted in the proof of Lemma 2.5, $g \in T P_{\omega}$ if and only if

$$
\int_{0}^{\omega} e^{-B\left(\alpha_{0}\right) s} \Psi_{\alpha_{0}}(0) g(s) d s=0 .
$$

So the bifurcation equation is equivalent to

$$
\int_{0}^{\omega} e^{-B\left(\alpha_{0}\right) s} \Psi_{\alpha_{0}}(0) N\left(\alpha, \beta, u_{x}^{*}(\alpha, \beta, z), u_{s, \beta}^{*}(\alpha, \beta, z)\right) d s=0 .
$$

Let $H: \mathbb{R}^{2 m} \rightarrow U_{i \beta_{0}}\left(A\left(\alpha_{0}\right)\right)$ denote the isomorphism defined in the proof of Lemma 2.4. Then there exists a vector $w_{1}=\left(w_{11}, w_{21}\right)^{T} \in \mathbb{R}^{2 m}, w_{11}, w_{12} \in \mathbb{R}^{m}$, such that $\left(\delta_{1}, \delta_{2}\right)=H\left(\begin{array}{cc}w_{11} & -w_{21} \\ w_{21} & w_{11}\end{array}\right)$. Define $G: \mathbb{R} \times \mathbb{R}_{+} \times \mathbb{R}^{2} \rightarrow \mathbb{R}^{2 m}$ by

$$
G(\alpha, \beta, z)=\int_{0}^{\omega} e^{-B\left(\alpha_{0}\right) s} \Psi_{\alpha_{0}}(0) N\left(\alpha, \beta, u_{s}^{*}(\alpha, \beta, z), u_{s, \beta}^{*}(\alpha, \beta, z)\right) d s .
$$

One can easily verify that

$$
\begin{aligned}
& (\gamma, \theta) G(z) \\
& \quad=\int_{0}^{\omega} e^{-B\left(\alpha_{0}\right)(s-\theta)} T_{g} \Psi_{\alpha_{0}}(0) N\left(\alpha, \beta, u_{s}^{*}(\alpha, \beta, z), u_{s, \beta}^{*}(\alpha, \beta, z)\right) d s \\
& \quad=\int_{0}^{\omega} e^{-B\left(\alpha_{0}\right)(s-\theta)} \Psi_{\alpha_{0}}(0) \gamma N\left(\alpha, \beta, u_{s}^{*}(\alpha, \beta, z), u_{s, \beta}^{*}(\alpha, \beta, z)\right) d s \\
& \quad=\int_{0}^{\omega} e^{-B\left(\alpha_{0}\right)(s-\theta)} \Psi_{\alpha_{0}}(0) N\left(\alpha, \beta, \gamma u_{s}^{*}(\alpha, \beta, z), \gamma u_{s, \beta}^{*}(\alpha, \beta, z)\right) d s \\
& =\int_{0}^{\omega} e^{-B\left(\alpha_{0}\right)(s-\theta)} \Psi_{\alpha_{0}}(0) N\left(\alpha, \beta, u_{s-\theta}^{*}(\alpha, \beta, z), u_{s-\theta, \beta}^{*}(\alpha, \beta, z)\right) d s \\
& =\int_{0}^{\omega} e^{-B\left(\alpha_{0}\right) s} \Psi_{\alpha_{0}}(0) N\left(\alpha, \beta, u_{s}^{*}(\alpha, \beta, z), u_{s, \beta}^{*}(\alpha, \beta, z)\right) d s \\
& =G(Z)
\end{aligned}
$$

for $(\gamma, \theta) \in \Sigma$. So $G\left(\mathbb{R} \times \mathbb{R}_{+} \times \mathbb{R}^{2}\right)$ is contained in the two-dimensional subspace of $\mathbb{R}^{2 m}$ spanned by $w_{1}=\left(w_{11}, w_{21}\right)^{T}$ and $w_{2}=\left(-w_{21}, w_{11}\right)^{T}$. Clearly, $G(\alpha, \beta, 0)=0$ and

$$
G(\alpha, \beta, z)=M(\alpha, \beta) z+o(|z|)
$$

where

$$
M(\alpha, \beta) z=\int_{0}^{\omega} e^{-B(\alpha) s} \Psi_{\alpha_{0}}(0)\left[(1+\beta) L(\alpha) v_{s, \beta}^{*}(\alpha, \beta)-L\left(\alpha_{0}\right) v_{s}^{*}(\alpha, \beta)\right] z d s
$$

and

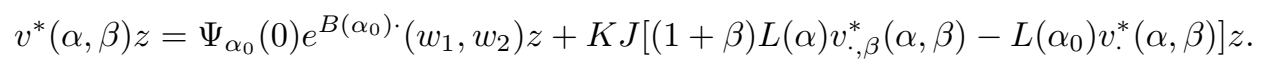

So,

$$
v^{*}\left(\alpha_{0}, \beta\right) z=\Psi_{\alpha_{0}}(0) e^{(1+\beta) B\left(\alpha_{0}\right) \cdot}\left(w_{1}, w_{2}\right) z
$$

Hence,

$$
\begin{aligned}
M(\alpha, \beta) z & =\beta \int_{0}^{\omega} e^{-B\left(\alpha_{0}\right) s} \Psi_{\alpha_{0}}(0) \Phi_{\alpha_{0}}(0) e^{B\left(\alpha_{0}\right) s} B\left(\alpha_{0}\right)\left(w_{1}, w_{2}\right) z d s \\
& =\beta \int_{0}^{\omega}\left(\Psi_{\alpha_{0}}, \Phi_{\alpha_{0}}\right)_{\alpha_{0}} B\left(\alpha_{0}\right)\left(w_{1}, w_{2}\right) z d s .
\end{aligned}
$$


Therefore,

$$
\begin{aligned}
\frac{\partial}{\partial \beta} M & \left.\left(\alpha_{0}, \beta\right) z\right|_{\beta=0} \\
& =\int_{0}^{\omega}\left(\Psi_{\alpha_{0}}, \Phi_{\alpha_{0}}\right)_{\alpha_{0}} B\left(\alpha_{0}\right)\left(w_{1}, w_{2}\right) z d s \\
& =\omega\left(\Psi_{\alpha_{0}}, \Phi_{\alpha_{0}}\right)_{\alpha_{0}} B\left(\alpha_{0}\right)\left(w_{1}, w_{2}\right) z \\
& =\left(w_{1}, w_{2}\right) \omega\left(\Psi_{\alpha_{0}}, \Phi_{\alpha_{0}}\right)_{\alpha_{0}}\left(\begin{array}{cc}
0 & \beta_{0} \\
-\beta_{0} & 0
\end{array}\right) z .
\end{aligned}
$$

Moreover, by Lemma 2.2, we have

$$
\begin{aligned}
\frac{\partial}{\partial \alpha} M & \left.\left(\alpha, \beta_{0}\right) z\right|_{\alpha=\alpha_{0}} \\
& =\int_{0}^{\omega} e^{-B\left(\alpha_{0}\right) s} \Psi_{\alpha_{0}} L\left(\alpha_{0}\right) \Phi_{\alpha_{0}} e^{B\left(\alpha_{0}\right) s}\left(w_{1}, w_{2}\right) z d s \\
& =-\int_{0}^{\omega} e^{-B\left(\alpha_{0}\right) s}\left(\Psi_{\alpha_{0}}, \Phi_{\alpha_{0}}\right)_{\alpha_{0}} B^{\prime}\left(\alpha_{0}\right) e^{B\left(\alpha_{0}\right) s}\left(w_{1}, w_{2}\right) z d s \\
& =-\left(w_{1}, w_{2}\right) \omega\left(\Psi_{\alpha_{0}}, \Phi_{\alpha_{0}}\right)_{\alpha_{0}}\left(\begin{array}{cc}
\operatorname{Re} \lambda^{\prime}\left(\alpha_{0}\right) & -\operatorname{Im} \lambda^{\prime}\left(\alpha_{0}\right) \\
\operatorname{Im} \lambda^{\prime}\left(\alpha_{0}\right) & \operatorname{Re} \lambda^{\prime}\left(\alpha_{0}\right)
\end{array}\right) z .
\end{aligned}
$$

Consequently, we get

$$
\begin{gathered}
{\left[\frac{\partial}{\partial \alpha} M(\alpha, \beta), \frac{\partial}{\partial \beta} M(\alpha, \beta)\right]_{\alpha=\alpha_{0}, \beta=\beta_{0}} z} \\
\quad=\omega\left(\begin{array}{cc}
-\operatorname{Re} \lambda^{\prime}\left(\alpha_{0}\right) & \beta+\operatorname{Im} \lambda^{\prime}\left(\alpha_{0}\right) \\
-\beta_{0}-\operatorname{Im} \lambda^{\prime}\left(\alpha_{0}\right) & \operatorname{Re} \lambda^{\prime}\left(\alpha_{0}\right)
\end{array}\right) z .
\end{gathered}
$$

As $G$ commutes with the $S^{1}$-action, we have

$$
\begin{aligned}
& G(\alpha, \beta, z)=M(\alpha, \beta) z+o(|z|) \\
& \quad=p(\alpha, \beta)\left(\begin{array}{c}
z_{1} \\
z_{2}
\end{array}\right)+q(\alpha, \beta)\left(\begin{array}{c}
-z_{2} \\
z_{1}
\end{array}\right)+o(|z|) .
\end{aligned}
$$

So, (2.22) implies that

$$
\begin{aligned}
& \left.\frac{\partial}{\partial \alpha} p\left(\alpha, \beta_{0}\right)\right|_{\alpha=\alpha_{0}}=-\omega \operatorname{Re} \lambda^{\prime}\left(\alpha_{0}\right), \\
& \left.\frac{\partial}{\partial \alpha} q\left(\alpha, \beta_{0}\right)\right|_{\alpha=\alpha_{0}}=\operatorname{Im} \lambda^{\prime}\left(\alpha_{0}\right), \\
& \left.\frac{\partial}{\partial \beta} p\left(\alpha_{0}, \beta\right)\right|_{\beta=\beta_{0}}=0, \\
& \left.\frac{\partial}{\partial \beta} q\left(\alpha_{0}, \beta\right)\right|_{\beta=\beta_{0}}=-\omega \beta_{0} .
\end{aligned}
$$

The remainder of the proof proceeds exactly as in the proof of the standard Hopf bifurcation theorem (cf. Hale [27]), and therefore is omitted.

\section{A topological global Hopf Bifurcation theorem}

Theorem 2.1 enables us to detect generic Hopf bifurcations of periodic solutions. It also describes the spatial-temporal symmetry of the bifurcated periodic solutions. Therefore, one can often obtain a reduced system of functional differential equations which characterizes the bifurcated periodic solutions. In particular, for 
the delayed neural network to be studied in subsequent sections, generic periodic solutions are discrete waves, mirror-reflecting waves and standing waves which are completely characterized by a scalar functional differential equation with the period as an additional parameter. Due to this additional parameter, the reduced scalar functional differential equations have several discrete delayed and advanced arguments. This motivates us to consider the local existence and global continuation of periodic solutions for functional differential equations of mixed type and with two parameters.

We will need the following $S^{1}$-bifurcation theory for a coincidence problem with two parameters. Let $\mathbb{E}$ be a real isometric Banach representation of the group $G=S^{1}$. The isotypical direct sum decomposition is denoted by

$$
\mathbb{E}=\mathbb{E}_{0} \oplus \mathbb{E}_{1} \oplus \cdots \oplus \mathbb{E}_{k} \oplus \cdots,
$$

where $\mathbb{E}_{0}=E^{G}:=\{x \in G ; g x=x$ for all $g \in G\}$ is the subspace of $G$-fixed points, and for $k \geq 1, x \in \mathbb{E}_{k} \backslash\{0\}$ implies $G_{x}$, the isotropy group of $x$, is $\mathbb{Z}_{k}:=$ $\left\{g \in G ; g^{k}=1\right\}$. For simplicity, we assume that each $\mathbb{E}_{k}, k=0,1, \ldots$, is of finite dimension.

All subspaces $\mathbb{E}_{k}, k \geq 1$, admit a natural structure of complex vector spaces such that an $\mathbb{R}$-linear operator $A: \mathbb{E}_{k} \rightarrow \mathbb{E}_{k}$ is $G$-equivariant if and only if it is $\mathbb{C}$-linear with respect to this complex structure. Therefore, by choosing a basis in $\mathbb{E}_{k}, k \geq 1$, we can define an isomorphism between the group of all $G$-equivariant automorphisms of $\mathbb{E}_{k}$, denoted by $G L_{G}\left(\mathbb{E}_{k}\right)$, and the general linear group $G L\left(m_{k}, \mathbb{C}\right)$, where $m_{k}=\operatorname{dim}_{\mathbb{C}} \mathbb{E}_{k}$.

For a topological space $X$, we denote by $\left[S^{1}, X\right]$ the set of homotopy classes of continuous maps $\beta: S^{1} \rightarrow X$. Let $\mathbb{C}^{*}:=\mathbb{C} \backslash\{0\}$ be a continuous map. The correspondence $[\beta] \rightarrow \operatorname{deg}_{B}(\beta)$, where $\operatorname{deg}_{B}$ denotes the Brouwer degree, defines the bijection of $\left[S^{1}, \mathbb{C}^{*}\right]$ onto $\mathbb{Z}$. It is well-known that there exists a canonical bijection $\nabla:\left[S^{1}, G L(n, \mathbb{C})\right] \rightarrow \mathbb{Z}$ defined by $\nabla([\alpha]):=\operatorname{deg}_{B}\left(\operatorname{det}_{\mathbb{C}} \alpha\right)$, where $\alpha: S^{1} \rightarrow G L(n, \mathbb{E})$ and $\operatorname{det}_{\mathbb{C}}: G L(n, \mathbb{C}) \rightarrow \mathbb{C}^{*}$ is the usual determinant homomorphism. Moreover, if $\alpha_{i}: S^{1} \rightarrow G L\left(n_{i}, \mathbb{C}\right), i=1,2$, are two continuous maps, then $\nabla\left(\left[\alpha_{1} \oplus \alpha_{2}\right]\right)=\nabla\left(\left[\alpha_{1}\right]\right) \cdot \nabla\left(\left[\alpha_{2}\right]\right)$, where $\alpha_{1} \oplus \alpha_{2}: S^{1} \rightarrow G L\left(n_{1}+n_{2}, \mathbb{C}\right)$ is the canonical direct sum of $\alpha_{1}$ and $\alpha_{2}$.

Let $\mathbb{F}$ be another Banach isometric representation of $G$, and $L: \mathbb{E} \rightarrow \mathbb{F}$ be a given equivariant linear bounded Fredholm operator of index zero. We assume that $L$ has an equivariant compact resolvent $K: \mathbb{E} \rightarrow \mathbb{F}$. That is, $K$ is equivariant and $L+K: \mathbb{E} \rightarrow \mathbb{F}$ is an isomorphism.

In what follows, a point of the Banach space $\mathbb{E} \times \mathbb{R}^{2}$ is denoted by $(x, \lambda)$ with $x \in \mathbb{E}$ and $\lambda \in \mathbb{R}^{2}$, and the action of $G$ on $\mathbb{E} \times \mathbb{R}^{2}$ is defined by $g(x, \lambda)=(g x, \lambda)$ for every $g \in G$.

We consider a $G$-equivariant continuous map $f: \mathbb{E} \times \mathbb{R}^{2} \rightarrow \mathbb{F}$ such that

$$
f(x, \lambda)=L x-Q(x, \lambda), \quad(x, \lambda) \in X \times \mathbb{R}^{2},
$$

where $Q: \mathbb{E} \times \mathbb{R}^{2} \rightarrow \mathbb{F}$ is a completely continuous map and the following assumption is satisfied

(B1) There exists a two-dimensional submanifold $N \subset \mathbb{E}_{0} \times \mathbb{R}^{2}$ such that (i) $N \subset f^{-1}(0)$; (ii) if $\left(x_{0}, \lambda_{0}\right) \in N$, then there exists an open neighbourhood $U_{\lambda_{0}}$ of $\lambda_{0}$ in $\mathbb{R}^{2}$, an open neighbourhood $U_{x_{0}}$ of $x_{0}$ in $\mathbb{E}_{0}$, and a $C^{1}$-map $\eta: U_{x_{0}} \rightarrow \mathbb{E}_{0}$ such that $N \cap\left(U_{x_{0}} \times U_{\lambda_{0}}\right)=\left\{(\eta(\lambda), \lambda) ; \lambda \in U_{\lambda_{0}}\right\}$. 
We further assume that at all points $\left(x_{0}, \lambda_{0}\right) \in N$ the derivative $D_{x} f\left(x_{0}, \lambda_{0}\right): \mathbb{E}$ $\rightarrow \mathbb{F}$ of $f$ with respect to $x$ exists and is continuous on $N$. We say that $\left(x_{0}, \lambda_{0}\right)$ $\in N$ is $\mathbb{E}$-singular if $D_{x} f\left(x_{0}, \lambda_{0}\right): \mathbb{E} \rightarrow \mathbb{F}$ is not an isomorphism. An $\mathbb{E}$-singular point $\left(x_{0}, \lambda_{0}\right)$ is isolated if there are no other $\mathbb{E}$-singular points in some neighbourhood of $\left(x_{0}, \lambda_{0}\right)$.

Suppose that $\left(x_{0}, \lambda_{0}\right) \in N$ is an isolated $\mathbb{E}$-singular point. We identify $\mathbb{R}^{2}$ with $\mathbb{C}$, and for sufficiently small $\rho>0$, we define $\alpha: D \rightarrow N, D:=\{z \in \mathbb{C} ;|z| \leq 1\}$, by

$$
\alpha(z)=\left(\eta\left(\lambda_{0}+\rho z\right), \lambda_{0}+\rho z\right) \in \mathbb{E}_{0} \times \mathbb{R}^{2} .
$$

Let

$$
h(x, \lambda)=x-(L+K)^{-1}(K x+Q(x, \lambda)), \quad(x, \lambda) \in X \oplus \mathbb{R}^{2} .
$$

Clearly, the formula $\Psi(z):=D_{x} h(\alpha(z)), z \in \partial D$, defines a continuous mapping $\Psi: S^{1} \rightarrow G L_{G}(\mathbb{E})$ which has the decomposition $\Psi=\Psi_{0} \oplus \Psi_{1} \oplus \cdots \oplus \Psi_{k} \oplus \cdots$, where $\Psi_{0}: S^{1} \rightarrow G L\left(\mathbb{E}_{0}\right)$ and $\Psi_{k}: S^{1} \rightarrow G L_{G}\left(\mathbb{E}_{k}\right)$ for $k=1,2, \ldots$ We now define

$$
\varepsilon=\operatorname{sign} \operatorname{det} \Psi_{0}(z), \quad z \in S^{1},
$$

and

$$
\gamma_{k}\left(x_{0}, \lambda_{0}\right)=\varepsilon \nabla\left(\left[\Psi_{k}\right]\right), \quad k=1,2, \ldots .
$$

Geba and Marzantowicz [21] established the following global bifurcation result by applying the $S^{1}$-degree theory due to Dylawerski, Geba, Jodel and Marzantowicz [12].

Theorem 3.1. Suppose that $f$ satisfies (B1). If $\left(x_{0}, \lambda_{0}\right) \in N$ is an isolated $\mathbb{E}$ singular point such that $\gamma_{k}\left(x_{0}, \lambda_{0}\right) \neq 0$ for some $k \geq 1$, then there exists a sequence $\left(x_{n}, \lambda_{n}\right) \in f^{-1}(0) \backslash N$ such that $\left(x_{n}, \lambda_{n}\right) \rightarrow\left(x_{0}, \lambda_{0}\right)$ as $n \rightarrow \infty$ and $\mathbb{Z}_{k} \subset G_{x_{n}}$ for each $n \geq 1$. Moreover, if we assume that $N$ is complete and every $\mathbb{E}$-singular point in $N$ is isolated, then for each bounded connected component $\mathcal{P}$ of $\mathcal{S}(f)$, where $\mathcal{S}(f)$ denotes the closure of the set $f^{-1}(0) \backslash N$, the set $\mathcal{P} \cap \mathcal{S}(f)$ is finite and

$$
\sum_{(x, \lambda) \in \mathcal{P} \cap \mathcal{S}(f)} \gamma_{k}(x, \lambda)=0
$$

for every positive integer $k$.

With the above preparation, we can now consider global Hopf bifurcations for general functional differential equations of mixed type with two parameters.

Let $X$ denote the Banach space of bounded continuous mappings $x: \mathbb{R} \rightarrow \mathbb{R}^{n}$ equipped with the supremum norm. For reasons discussed at the beginning of this section, we will consider functional differential equations with both delayed and advanced arguments. Therefore, for $x \in X$ and $t \in \mathbb{R}$, we will use $x^{t}$ to denote an element in $X$ defined by $x^{t}(s)=x(t+s)$ for $s \in \mathbb{R}$.

Consider the following functional differential equation

$$
\dot{x}(t)=F\left(x^{t}, \alpha, p\right)
$$

parametrized by two real numbers $(\alpha, p) \in \mathbb{R} \times \mathbb{R}_{+}$, where $\mathbb{R}_{+}=(0, \infty)$ and $F: X \times$ $\mathbb{R} \times R_{+} \rightarrow R^{n}$ is completely continuous. Identifying the subspace of $X$ consisting of all constant mappings with $\mathbb{R}^{n}$, we obtain a mapping $\widehat{F}=\left.F\right|_{\mathbb{R}^{n} \times \mathbb{R} \times \mathbb{R}_{+}}: \mathbb{R}^{n} \times \mathbb{R} \times$ $\mathbb{R}_{+} \rightarrow \mathbb{R}^{n}$. We require

(A1) $\widehat{F}$ is twice continuously differentiable. 
Denote by $\hat{x}_{0} \in X$ the constant mapping with the value $x_{0} \in \mathbb{R}^{n}$. We call $\left(\hat{x}_{0}, \alpha_{0}, p_{0}\right)$ a stationary solution of $(3.1)$ if $\widehat{F}\left(x_{0}, \alpha_{0}, p_{0}\right)=0$. We assume

(A2) At each stationary solution $\left(\hat{x}_{0}, \alpha_{0}, p_{0}\right)$, the derivative of $\widehat{F}(x, \alpha, p)$ with respect to the first variable $x$, evaluated at $\left(\hat{x}_{0}, \alpha_{0}, p_{0}\right)$, is an isomorphism of $\mathbb{R}^{n}$

Under (A1)-(A2), for each stationary solution $\left(\hat{x}_{0}, \alpha_{0}, p_{0}\right)$ there exists $\varepsilon_{0}>0$ and a continuously differentiable mapping $y: B_{\varepsilon_{0}}\left(\alpha_{0}, p_{0}\right) \rightarrow R^{n}$ such that $\widehat{F}(y(\alpha, p), \alpha, p)$ $=0$ for $(\alpha, p) \in B_{\varepsilon_{0}}\left(\alpha_{0}, p_{0}\right)=\left(\alpha_{0}-\varepsilon_{0}, \alpha_{0}+\varepsilon_{0}\right) \times\left(p_{0}-\varepsilon_{0}, p_{0}+\varepsilon_{0}\right)$.

We need the following smoothness condition:

(A3) $F(\varphi, \alpha, p)$ is differentiable with respect to $\varphi$, and the $n \times n$ complex matrix function $\Delta_{(\hat{y}(\alpha, p), \alpha, p)}(\lambda)$ is continuous in $(\alpha, p, \lambda) \in B_{\varepsilon_{0}}\left(\alpha_{0}, p_{0}\right) \times \mathbb{C}$. here, for each stationary solution $\left(\hat{x}_{0}, \alpha_{0}, p_{0}\right)$, we have $\Delta_{\left(\hat{x}_{0}, \alpha_{0}, p_{0}\right)}(\lambda)=\lambda \mathrm{Id}-$ $D F\left(\hat{x}_{0}, \alpha_{0}, p_{0}\right)\left(e^{\lambda \cdot} \mathrm{Id}\right)$, where $D F\left(\hat{x}_{0}, \alpha_{0}, p_{0}\right)$ is the complexification of the derivative of $F(\varphi, \alpha, p)$ with respect to $\varphi$, evaluated at $\left(\hat{x}_{0}, \alpha_{0}, p_{0}\right)$.

For easy reference, we will again call $\Delta_{\left(\hat{x}_{0}, \alpha_{0}, p_{0}\right)}(\lambda)$ the characteristic matrix and the zeros of $\operatorname{det} \Delta_{\left(\hat{x}_{0}, \alpha_{0}, p_{0}\right)}(\lambda)=0$ the characteristic values of the stationary solution $\left(\hat{x}_{0}, \alpha_{0}, p_{0}\right)$. So, (A2) is equivalent to assuming that 0 is not a characteristic value of any stationary solution of (3.1).

Definition 3.1. A stationary solution $\left(\hat{x}_{0}, \alpha_{0}, p_{0}\right)$ is called a center if it has purely imaginary characteristic values of the form $i m \frac{2 \pi}{p_{0}}$ for some positive integer $m$. A center $\left(\hat{x}_{0}, \alpha_{0}, p_{0}\right)$ is said to be isolated if (i) it is the only center in some neighborhood of $\left(\hat{x}_{0}, \alpha_{0}, p_{0}\right)$; (ii) it has only finitely many purely imaginary characteristic values of the form $i m \frac{2 \pi}{p_{0}}, m$ is an integer.

Assume now $\left(\hat{x}_{0}, \alpha_{0}, p_{0}\right)$ is an isolated center. Let $J\left(\hat{x}_{0}, \alpha_{0}, p_{0}\right)$ denote the set of all positive integers $m$ such that $i m \frac{2 \pi}{p_{0}}$ is a characteristic value of $\left(\hat{x}_{0}, \alpha_{0}, p_{0}\right)$. We assume that there exists $m \in J\left(\hat{x}_{0}, \alpha_{0}, p_{0}\right)$ such that

(A4) There exist $\varepsilon \in\left(0, \varepsilon_{0}\right)$ and $\delta \in\left(0, \varepsilon_{0}\right)$ so that on $\left[\alpha_{0}-\delta, \alpha_{0}+\delta\right] \times \partial \Omega_{\varepsilon, p_{0}}$, $\operatorname{det} \Delta_{(\hat{y}(\alpha, p), \alpha, p)}\left(u+i m \frac{2 \pi}{p}\right)=0$ if and only if $\alpha=\alpha_{0}, u=0, p=p_{0}$, where $\Omega_{\varepsilon, p_{0}}=\left\{(u, p) ; 0<u<\varepsilon, p_{0}-\varepsilon<p<p_{0}+\varepsilon\right\}$.

Let

$$
H^{ \pm}\left(\hat{x}_{0}, \alpha_{0}, p_{0}\right)(u, p)=\operatorname{det} \Delta_{\left(\hat{y}\left(\alpha_{0} \pm \delta, p\right), \alpha_{0} \pm \delta, p\right)}\left(u+i m \frac{2 \pi}{p}\right) .
$$

Then (A4) implies that $H_{m}^{ \pm}\left(\hat{x}_{0}, \alpha_{0}, p_{0}\right) \neq 0$ on $\partial \Omega_{\varepsilon, p_{0}}$. Consequently, the following integer

$$
\gamma_{m}\left(\hat{x}_{0}, \alpha_{0}, p_{0}\right)=\operatorname{deg}_{B}\left(H_{m}^{-}\left(\hat{x}_{0}, \alpha_{0}, p_{0}\right), \Omega_{\varepsilon, p_{0}}\right)-\operatorname{deg}_{B}\left(H_{m}^{+}\left(\hat{x}_{0}, \alpha_{0}, p_{0}\right), \Omega_{\varepsilon, p_{0}}\right)
$$

is well defined.

Definition 3.2. $\gamma_{m}\left(\hat{x}_{0}, \alpha_{0}, p_{0}\right)$ is called the $m$ th crossing number of $\left(\hat{x}_{0}, \alpha_{0}, p_{0}\right)$.

We will show that $\gamma_{m}\left(\hat{x}_{0}, \alpha_{0}, p_{0}\right) \neq 0$ implies the existence of a local bifurcation of periodic solutions with periods near $p_{0} / \mathrm{m}$. More precisely, we have the following:

Theorem 3.2. Assume that (A1)-(A3) are satisfied, and that there exists an isolated center $\left(\hat{x}_{0}, \alpha_{0}, p_{0}\right)$ and an integer $m \in J\left(\hat{x}_{0}, \alpha_{0}, p_{0}\right)$ such that (A4) holds and $\gamma_{m}\left(\hat{x}_{0}, \alpha_{0}, p_{0}\right) \neq 0$. Then there exists a sequence $\left(\alpha_{k}, p_{k}\right) \in \mathbb{R} \times \mathbb{R}_{+}$so that

(i) $\lim _{k \rightarrow \infty}\left(\alpha_{k}, p_{k}\right)=\left(\alpha_{0}, p_{0}\right)$; 
(ii) at each $(\alpha, p)=\left(\alpha_{k}, p_{k}\right)$, (3.1) has a non-constant periodic solution $x_{k}(t)$ with a period $p_{k} / m$.

(iii) $\lim _{k \rightarrow \infty} x_{k}(t)=\hat{x}_{0}$, uniformly for $t \in \mathbb{R}$.

To describe the global continuation of the local bifurcation obtained in Theorem 3.2, we need to assume

(H5) All centers of (3.1) are isolated and (A4) holds for each center $\left(\hat{x}_{0}, \alpha_{0}, p_{0}\right)$ and each $m \in J\left(\hat{x}_{0}, \alpha_{0}, p_{0}\right)$.

(H6) For each bounded set $W \subseteq X \times \mathbb{R} \times \mathbb{R}_{+}$there exists a constant $L>0$ such that $|F(\varphi, \alpha, p)-F(\psi, \alpha, p)| \leq L \sup _{s \in R}|\varphi(s)-\psi(s)|$ for $(\varphi, \alpha, p),(\psi, \alpha, p) \in$ $W$.

Theorem 3.3. Let

$$
\begin{aligned}
\Sigma(F) & =C l\{(x, \alpha, p) ; x \text { is a p-periodic solution of }(3.1)\} \subset X \times \mathbb{R} \times \mathbb{R}, \\
N(F) & =\{(\hat{x}, \alpha, p) ; F(\hat{x}, \alpha, p)=0\} .
\end{aligned}
$$

Assume that $\left(\hat{x}_{0}, \alpha_{0}, p_{0}\right)$ is an isolated center satisfying conditions in Theorem 3.2. Denote by $C\left(\hat{x}_{0}, \alpha_{0}, p_{0}\right)$ the connected component of $\left(\hat{x}_{0}, \alpha_{0}, p_{0}\right)$ in $\Sigma(F)$. Then either

(i) $C\left(\hat{x}_{0}, \alpha_{0}, p_{0}\right)$ is unbounded, or

(ii) $C\left(\hat{x}_{0}, \alpha_{0}, p_{0}\right)$ is bounded, $C\left(\hat{x}_{0}, \alpha_{0}, p_{0}\right) \cap N(F)$ is finite and

$$
\sum_{(\hat{x}, \alpha, p) \in C\left(\hat{x}_{0}, \alpha_{0}, p_{0}\right) \cap N(F)} \gamma_{m}(\hat{x}, \alpha, p)=0
$$

for all $m=1,2, \ldots$, where $\gamma_{m}(\hat{x}, \alpha, p)$ is the mth crossing number of $(\hat{x}, \alpha, p)$ if $m \in J(\hat{x}, \alpha, p)$, or it is zero if otherwise.

Proof of Theorems 3.2 and 3.3. Put $S^{1}=\mathbb{R} / 2 \pi \mathbb{Z}, \mathbb{E}=L^{1}\left(S^{1} ; \mathbb{R}^{n}\right), M=L^{2}\left(S^{1} ; \mathbb{R}^{n}\right)$. Define $L: \mathbb{E} \rightarrow M$ and $Q: \mathbb{E} \times \mathbb{R} \times \mathbb{R}_{+} \rightarrow M$ by

$$
L z=\dot{z}(t), Q(z, \alpha, p)(t)=\frac{p}{2 \pi} F\left(z_{t, p}, \alpha, p\right),
$$

where

$$
z_{t, p}(\theta)=z\left(t+\frac{2 \pi}{p} \theta\right), \theta \in R
$$

Clearly, $x(t)$ is a $p$-periodic solution of (3.1) if and only if $z(t)=x\left(\frac{p}{2 \pi} t\right)$ is a solution in $\mathbb{E}$ of the operator equation $L z=Q(z, \alpha, p)$.

$\mathbb{E}$ and $\mathbb{F}$ are isometric Hilbert representations of the group $S^{1}$, where $S^{1}$ acts by shifting the argument. With respect to these $S^{1}$-actions, $L$ is an equivariant bounded linear Fredholm operator of index zero with an equivariant compact resolvent $K$, and $Q$ is an $S^{1}$-equivariant compact mapping. Moreover, at $(\hat{y}(\alpha, p), \alpha, p)$ with $(\alpha, p) \in \mathcal{D}:=\left(\alpha_{0}-\delta, \alpha_{0}+\delta\right) \times\left(p_{0}-\varepsilon, p_{0}+\varepsilon\right)$, the derivative of $Q$ with respect to the first variable is given by

$$
D_{z} Q(\hat{y}(\alpha, p), \alpha, p) z(t)=\frac{p}{2 \pi} D F(\hat{y}(\alpha, p), \alpha, p) z_{t, p} .
$$

Identifying $\partial \mathcal{D}$ with $S^{1}$, as $\left(\hat{x}_{0}, \alpha_{0}, p_{0}\right)$ is an isolated center we can easily show that the mapping $\operatorname{Id}-(L+K)^{-1}\left[K+D_{z} F(\hat{y}(\alpha, p), \alpha, p)\right]$ is an isomorphism of $\mathbb{E}$ and that the mapping $\Psi: S^{1} \rightarrow G L(E)$ defined by

$$
(\alpha, p) \in \partial \mathcal{D} \cong S^{1} \rightarrow \mathrm{Id}-(L+K)^{-1}\left[K+D_{z} F(\hat{y}(\alpha, p), \alpha, p)\right] \in G L(\mathbb{E})
$$

is continuous. 
$\mathbb{E}$ has the well-known isotypical decomposition $\mathbb{E}=\bigoplus_{k=0}^{\infty} \mathbb{E}_{k}$, where $\mathbb{E}_{0} \cong \mathbb{R}^{n}$ and for each $k \geq 1, E_{k}$ is spanned by $\cos (k t) \varepsilon_{j}$ and $\sin (k t) \varepsilon_{j}, 1 \leq j \leq n$, where $\left\{\varepsilon_{1}, \ldots, \varepsilon_{n}\right\}$ is the standard basis of $\mathbb{R}^{n}$. So, we have $\Psi(\alpha, p) \mathbb{E}_{k} \subseteq \mathbb{E}_{k}$. Let $\Psi_{k}(\alpha, p)=\left.\Psi(\alpha, p)\right|_{\mathbb{E}_{k}}$. It is not difficult to show that

$$
\Psi_{k}(\alpha, p)=\frac{p}{i 2 k \pi} \Delta_{(\hat{y}(\alpha, p), \alpha, p)}\left(i k \frac{2 \pi}{p}\right) .
$$

Let

$$
\begin{gathered}
\varepsilon=\operatorname{sign} \operatorname{det} \Psi_{0}(\alpha, p), \quad(\alpha, p) \in \partial \mathcal{D} . \\
n_{k}\left(\hat{x}_{0}, \alpha_{0}, p_{0}\right)=\varepsilon \operatorname{deg}_{B}\left(\operatorname{det} \Psi_{k}(\cdot), \mathcal{D}\right), \quad k=1,2, \ldots .
\end{gathered}
$$

Then one can show, as in Erbe, Geba, Krawcewicz and Wu [13], that $\gamma_{k}\left(\hat{x}_{0}, \alpha_{0}, p_{0}\right)=$ $n_{k}\left(\hat{x}_{0}, \alpha_{0}, p_{0}\right)$ and therefore Theorems 3.2 and 3.3 are simply an immediate consequence of Theorem 3.1 with $N=\left\{\left(\hat{x}_{0}, \alpha_{0}, p_{0}\right) \in \mathbb{R}^{n} \times \mathbb{R} \times \mathbb{R}_{+} ; F\left(\hat{x}_{0}, \alpha_{0}, p_{0}\right)=0\right\}$. This completes the proof.

\section{Applications to Delayed neural networks:}

LOCAL EXISTENCE AND ASYMPTOTIC FORMS OF WAVES

We now consider the following system of delay-differential equations

$$
C_{i} \dot{u}_{i}(s)=-\frac{1}{R_{i}} u_{i}(s)+\sum_{j=1}^{n} T_{i j} f_{j}\left(u_{j}\left(s-\tau_{j}\right)\right), \quad 1 \leq i \leq n,
$$

which describes the evolution of a network of $n$ saturating voltage amplifiers (neurons) with delayed output coupled via a resistive interconnection matrix, where the variable $u_{i}$ represents the voltage on the input of the $i$ th neuron, and each neuron is characterized by an input capacitance $C_{i}$, and a transfer function $f_{i}$ which is sigmoidal, saturating at \pm 1 with maximum slope at $u=0$. More precisely, the transfer function $f_{i}$ satisfies the following condition:

(TF) $f_{i}: \mathbb{R} \rightarrow \mathbb{R}$ is twicely continuously differentiable, strictly increasing, $f(0)=$ $0, \lim _{x \rightarrow \pm \infty} f_{i}(x)= \pm 1$ and $x f_{i}^{\prime \prime}(x)<0$ if $x \neq 0$.

$T=\left(T_{i j}\right)$ is called the interconnection matrix where $T_{i j}$ has a value $\left(R_{i j}\right)^{-1}$ when the noninverting output of the $j$ th neuron is connected to the input of the $i$ th neuron through a resistance $R_{i j}$, and a value $-\left(R_{i j}\right)^{-1}$ when the inverting output of the $j$ th neuron is connected to the input of the $i$ th neuron through a resistance $R_{i j}$. $R_{i}=\left(\sum_{j=1}^{n}\left|T_{i j}\right|\right)^{-1}$ is called the parallel resistance at the input of the $i$ th neuron. The system was proposed by Hopfield $[33,34]$ and the time delay was incorporated by Marcus and Westervelt [47] to account for the finite switching speed of amplifiers. Similar systems were also investigated by Cohen and Grossberg [10].

We will concentrate on the case of identical neurons $C_{i}=C, \tau_{i}=\tau^{*}, f_{i}=f$, $R_{i}=R, 1 \leq i \leq n$. Rescaling time, delay and $T_{i j}$ by

$$
t=s / R C, \quad \tau=\tau^{*} / R C, \quad J_{i j}=R T_{i j},
$$

we obtain the following normalized system

$$
\dot{u}_{i}(t)=-u_{i}(t)+\sum_{j=1}^{n} J_{i j} f\left(u_{j}(t-\tau)\right), \quad 1 \leq i \leq n .
$$


Clearly, $J_{i j}$ has the following normalization property

$$
\sum_{j=1}^{n}\left|J_{i j}\right|=1 .
$$

In what follows, $J=\left(J_{i j}\right)$ will be called the normalized interconnection matrix, and $R C$ the characteristic network relaxation time.

We further assume that the normalized interconnection matrix $J$ is a symmetric circulant matrix, i.e. $J_{i j}=a_{j-i+1}$, where $a_{i}=a_{n-i+2}$ and the subscripts $a_{k}$ are written modulo $n$, so $a_{0}=a_{n}, a_{-1}=a_{n-1}$, etc. We will denote this by

$$
J_{i j}=\operatorname{circ}\left(a_{1}, a_{2}, \ldots, a_{n}\right) .
$$

Circulant interconnection matrix includes the following four important special cases:

$$
\begin{aligned}
J_{E} & =\frac{1}{n-1} \operatorname{circ}(0,1, \ldots, 1), \\
J_{I} & =\frac{1}{n-1} \operatorname{circ}(0,-1, \ldots,-1), \\
J_{R E} & =\frac{1}{2} \operatorname{circ}(0,1,0,0, \ldots, 0,1), \\
J_{I E} & =\frac{1}{2} \operatorname{circ}(0,-1,0,0, \ldots, 0,-1)
\end{aligned}
$$

which represents all-excitatory or ferromagnetic networks, all-inhibitory or antiferomagnetic networks, symmetrically connected excitatory rings and symmetrically connected inhibitory rings of neurons, respectively.

We will consider the Hopf bifurcation of periodic solutions of (4.1) with a circulant interconnection matrix $J=\operatorname{circ}\left(a_{1}, \ldots, a_{n}\right)$. The linearization of $(4.1)$ at the trivial solution leads to

$$
\dot{u}_{i}(t)=-u_{i}(t)+\beta \sum_{j=1}^{n} J_{i j} u_{j}(t-\tau), \quad 1 \leq i \leq n,
$$

here

$$
\beta=f^{\prime}(0)
$$

is called the neuron gain. Regarding $\tau$ as the parameter, we first determine when the infinitesimal generator $A_{\tau}$ of (4.3) has a pair of purely imaginary eigenvalues. Some version of the following result has appeared in Belair [6] and Braddock and van den Driessche [7], we include the proof for the completeness of the presentation.

Lemma 4.1. Let $\gamma=\operatorname{Re}^{i \theta}, 0 \leq \theta<2 \pi$, and consider

$$
q(\lambda)=\lambda+1-\gamma e^{-\lambda \tau} .
$$

(i) If $R \leq 1$, then $q(\lambda)$ has no purely imaginary zeros for all $\tau \geq 0$;

(ii) If $R>1$, then for any integer $k$ such that $\tau_{k}:=\left(\theta-\arccos \frac{1}{R}+2 k \pi\right) / \sqrt{R^{2}-1}$ $>0, q(\lambda)$ has one and only one pair of purely imaginary zeros $\pm i \sqrt{R^{2}-1}$ if $\tau=\tau_{k}$, and has no pair of purely imaginary zeros if $0<\tau \neq \tau_{k}$ for all such $k$ 's;

(iii) If $R>1$ and $\tau_{k}>0$ for some $k$, then there exist a sufficiently small $\delta>0$ and a smooth curve $\lambda:\left(\tau_{k}-\delta, \tau_{k}+\delta\right) \rightarrow \mathbb{C}$ such that $q(\lambda(\tau))=0$ for all $\tau \in\left(\tau_{k}-\delta, \tau_{k}+\delta\right), \lambda\left(\tau_{k}\right)=i \sqrt{R^{2}-1}$ and $\left.\frac{d}{d \lambda} \operatorname{Re} \lambda(\tau)\right|_{\tau=\tau_{k}}>0$. 
Proof. For any $x>0$, we have

$$
\begin{aligned}
q(i x) & =i x+1-\operatorname{Re}^{i(\theta-\tau x)} \\
& =\sqrt{1+x^{2}} e^{i \arctan x}-\operatorname{Re}^{i(\theta-\tau x)} .
\end{aligned}
$$

So, $q(i x)=0$ if and only if

$$
\left\{\begin{array}{l}
\sqrt{1+x^{2}}=R, \\
\arctan x=\theta-\tau x+2 k \pi \text { for some integer } k
\end{array}\right.
$$

from which the conclusions (i) and (ii) follow.

As

$$
\begin{aligned}
\left.\frac{\partial}{\partial \lambda} q(\lambda)\right|_{\lambda=i \sqrt{R^{2}-1}, \tau=\tau_{k}} \\
=1+\left.r \tau e^{-\lambda \tau}\right|_{\lambda=i \sqrt{R^{2}-1}, \tau=\tau_{k}} \\
=1+\tau_{k}\left(i \sqrt{R^{2}-1}+1\right) \neq 0,
\end{aligned}
$$

there exist $\delta>0$ and a smooth curve $\lambda:\left(\tau_{k}-\delta, \tau_{k}+\delta\right) \rightarrow \mathbb{C}$ such that $q(\lambda(\tau))=0$ and $\lambda\left(\tau_{k}\right)=i \sqrt{R^{2}-1}$. Differentiating $q(\lambda(\tau))=0$ with respect to $\tau$, we get

$$
\lambda^{\prime}\left(\tau_{k}\right)=\frac{-\gamma \lambda\left(\tau_{k}\right) e^{-\lambda\left(\tau_{k}\right) \tau_{k}}}{1+\tau_{k} \gamma e^{-\lambda\left(\tau_{k}\right) \tau_{k}}}=\frac{-\lambda\left(\tau_{k}\right)\left[\lambda\left(\tau_{k}\right)+1\right]}{1+\tau_{k}\left[\lambda\left(\tau_{k}\right)+1\right]} .
$$

Therefore,

$$
\operatorname{Re} \lambda^{\prime}\left(\tau_{k}\right)=\frac{R^{2}-1}{\left(1+\tau_{k}\right)^{2}+\tau_{k}^{2}\left(R^{2}-1\right)}>0 .
$$

This completes the proof.

To apply the previous lemma to system (4.3), we put

$$
\begin{gathered}
\xi=e^{i \frac{2 \pi}{n}} \\
W_{r}=\left(1, \xi^{r}, \ldots, \xi^{(n-1) r}\right)^{T}, 0 \leq r \leq n-1 .
\end{gathered}
$$

Clearly, $\left\{W_{0}, \ldots, W_{n-1}\right\}$ spans $\mathbb{C}^{n}$. The eigenvalues of $A_{\tau}$ of (4.3) are determined by the equation

$$
\operatorname{det} \Delta(\tau, \lambda)=0
$$

where

$$
\Delta(\tau, \lambda)=(\lambda+1) \mathrm{Id}-\beta e^{-\lambda \tau} J
$$

Note that

$$
\begin{aligned}
\left(\Delta(\tau, \lambda) W_{r}\right)_{i} & =(\lambda+1) \xi^{(i-1) r}-\beta e^{-\lambda \tau} \sum_{j=1}^{n} J_{i j} \xi^{(j-1) r} \\
& =\left[\lambda+1-\beta e^{-\lambda \tau} \sum_{j=1}^{n} a_{j-i+1} \xi^{(j-i) r}\right] \xi^{(i-1) r} \\
& =\left(\lambda+1-\alpha_{r} \beta e^{-\lambda \tau}\right) \xi^{(i-1) r}
\end{aligned}
$$

where

$$
\alpha_{r}=\sum_{k=0}^{n-1} a_{k+1} \xi^{k r}
$$


So,

$$
\Delta(\tau, \lambda) W_{r}=\left(\lambda+1-\alpha_{r} \beta e^{-\lambda \tau}\right) W_{r}
$$

and hence,

$$
\operatorname{det} \Delta(\tau, \lambda)-\prod_{r=0}^{n-1}\left(\lambda+1-\alpha_{r} \beta e^{-\lambda \tau}\right) .
$$

Applying Lemma 4.1 to each factor of the above product, we get

Lemma 4.2. Assume that there exists $r \in\{0, \ldots, n-1\}$ such that $\left|\alpha_{r} \beta\right|>1$. Let

$$
\begin{aligned}
\alpha_{r} & =\left|\alpha_{r}\right| e^{i \theta_{r}}, 0 \leq \theta_{r}<2 \pi, \\
\tau_{r, k} & =\frac{1}{\sqrt{\left|\alpha_{r} \beta\right|^{2}-1}}\left[\theta_{r}-\arccos \frac{1}{\left|\alpha_{r} \beta\right|}+2 k \pi\right], k=0, \pm 1, \pm 2, \ldots
\end{aligned}
$$

Then

(i) For each $k=0, \pm 1, \pm 2, \ldots$ such that $\tau_{r, k}>0$, the generator of (4.3) has a pair of purely imaginary eigenvalues $\pm i \sqrt{\left|\alpha_{r} \beta\right|^{2}-1}$ and the corresponding generalized eigenspace $U_{i \sqrt{\left|\alpha_{r} \beta\right|^{2}-1}}\left(A_{\tau_{r, k}}\right)$ consists of vectors $\operatorname{Re}\left(e^{\left.i \sqrt{\left|\alpha_{r} \beta\right|-1} \cdot b\right)}\right.$ and $\operatorname{Im}\left(e^{i \sqrt{\left|\alpha_{r} \beta\right|^{2}-1} \cdot b}\right)$ such that $b \in \operatorname{ker} \Delta\left(\tau_{r, k}, i \sqrt{\left|\alpha_{r} \beta\right|^{2}-1}\right)$;

(ii) For each $k=0, \pm 1, \pm 2, \ldots$ such that $\tau_{r, k}>0$, there exist $\delta>0$ and $a$ smooth function $\lambda:\left(\tau_{r, k}-\delta, \tau_{r, k}+\delta\right) \rightarrow \mathbb{C}$ such that $\operatorname{det} \Delta(\tau, \lambda(\tau))=0$, $\operatorname{dim} U_{\lambda(\tau)}\left(A_{\tau}\right)=\operatorname{dim} U_{i \sqrt{\left|\alpha_{r} \beta\right|^{2}-1}}\left(A_{\tau_{r, k}}\right)$ for $\tau \in\left(\tau_{r, k}-\delta, \tau_{r, k}+\delta\right), \lambda\left(\tau_{r, k}\right)=$ $i \sqrt{R^{2}-1}$ and $\frac{d}{d r} \operatorname{Re} \lambda(\tau)>0$ at $\tau=\tau_{r, k}$.

We now explore the symmetry in system (4.1). Let $\Gamma=\mathbb{D}_{n}$ be the dihedral group of order $2 n$. $\Gamma$ acts on $\mathbb{R}^{n}$ by

$$
(\rho x)_{j}=x_{j-1},(\kappa x)_{j}=x_{n-j}, j(\bmod n),
$$

where $\rho$ is the generator of the cyclic subgroup $\mathbb{Z}_{n}$ and $\kappa$ is the flip. Clearly, system (4.1) is $\Gamma$-equivariant. It is an easy exercise to verify the following:

Lemma 4.3. Assume that there exists $r \in\left\{0, \ldots,\left[\frac{n}{2}\right]\right\}$ such that $\left|\alpha_{r} \beta\right|>1$ and $\alpha_{j} \neq \alpha_{r}$ for all $0 \leq j \neq r \leq\left[\frac{n}{2}\right]$.

(i) If $2 r \neq 0, n$, then $\operatorname{dim} \operatorname{ker} \Delta\left(\tau_{r, k}, i \sqrt{\left|\alpha_{r} \beta\right|^{2}-1}\right)=4$ (as a real vector space) for any integer $k$ such that $\tau_{r, k}>0$. Moreover, there exists a 2-dimensional absolutely irreducible representation $\mathbb{R}^{2}$ of $\Gamma$ such that $\operatorname{ker} \Delta\left(\tau_{r, k}, i \sqrt{\left|\alpha_{r} \beta\right|^{2}-1}\right)$ is $\Gamma$-isomorphic to $\mathbb{R}^{2} \oplus \mathbb{R}^{2}$;

(ii) If $r=0$ or $\frac{n}{2}$ (in the later case, $n$ must be even), then

$$
\operatorname{dim} \operatorname{ker} \Delta\left(\tau_{r, k}, i \sqrt{\left|\alpha_{r} \beta\right|^{2}-1}\right)=2
$$

(as a real vector space) for any integer $k$ such that $\tau_{r, k}>0$.

Assume now that $\left|\alpha_{r} \beta\right|>1$ for some $r \in\left\{0, \ldots,\left[\frac{n}{2}\right]\right\}$. Let $\omega=\frac{2 \pi}{\sqrt{\left|\alpha_{r} \beta\right|^{2}-1}}$, and define

$$
\begin{gathered}
P_{\omega}=\text { the Banach space of all continuous } \omega \text {-periodic mappings } \\
\text { from } \mathbb{R} \text { into } \mathbb{R}^{n} \text {, equipped with the supremum norm; }
\end{gathered}
$$




$$
\begin{aligned}
& S P_{\omega}=\text { the subspace of } P_{\omega} \text { consisting of all } \omega \text {-periodic solutions } \\
& \text { of (4.3), where } \tau=\tau_{r, k} .
\end{aligned}
$$

Clearly, for any $\theta \in(0, \omega)$,

$$
\Sigma^{\theta}=\left\{\left(\rho^{j}, e^{i \frac{2 \pi}{\omega} \theta_{j}}\right) ; 0 \leq j \leq n-1\right\}
$$

is a subgroup of $\Gamma \times S^{1}$. We first consider

$$
\begin{aligned}
& \operatorname{Fix}\left(\sigma^{\theta}, S P_{\omega}\right)=\left\{x \in S P_{\omega} ; \rho x(t)=x(t-\theta), t \in \mathbb{R}\right\} \\
& \quad=\left\{x \in S P_{\omega} ; x_{i-1}(t)=x_{i}(t-\theta), t \in \mathbb{R}, i(\bmod n)\right\} .
\end{aligned}
$$

Lemma 4.4. Assume that $\left|\alpha_{r} \beta\right|>1$ for some $r \in\left\{0, \ldots,\left[\frac{n}{2}\right]\right\}$, and $\alpha_{j} \neq \alpha_{r}$ for all $0 \leq j \neq r \leq\left[\frac{n}{2}\right]$. Then

$$
\operatorname{Fix}\left(\Sigma^{\theta}, S P_{\omega}\right)=\left\{\begin{array}{l}
\left\{x_{1} \varepsilon_{1}+x_{2} \varepsilon_{2} ; x_{1}, x_{2} \in \mathbb{R}\right\} \quad \text { if } \theta=\frac{r}{n} \omega, \\
\left\{x_{3} \varepsilon_{3}+x_{4} \varepsilon_{4} ; x_{3}, x_{4} \in \mathbb{R}\right\} \quad \text { if } \theta=\frac{n-r}{n} \omega, \\
\{0\} \quad \text { if } \theta \notin\left\{\frac{r}{n} \omega, \frac{n-r}{n} \omega\right\}
\end{array}\right.
$$

where

$$
\left\{\begin{array}{l}
\varepsilon_{1}=\cos ^{\omega} \operatorname{Re} W_{r}-\sin ^{\omega} \operatorname{Im} W_{r} \\
\varepsilon_{2}=\sin ^{\omega} \operatorname{Re} W_{r}+\cos ^{\omega} \operatorname{Im} W_{r} \\
\varepsilon_{3}=\cos ^{\omega} \operatorname{Re} W_{r}+\sin ^{\omega} \operatorname{Im} W_{r} \\
\varepsilon_{4}=\sin ^{\omega} \operatorname{Re} W_{r}-\cos ^{\omega} \operatorname{Im} W_{r}
\end{array}\right.
$$

and $\cos ^{\omega} \in P_{\omega}, \sin ^{\omega} \in P_{\omega}$ and defined by

$$
\left\{\begin{array}{l}
\cos ^{\omega} t=\cos \left(\frac{2 \pi}{\omega} t\right) \\
\sin ^{\omega} t=\sin \left(\frac{2 \pi}{\omega} t\right), t \in \mathbb{R}
\end{array}\right.
$$

In particular,

$$
\operatorname{dim} \operatorname{Fix}\left(\Sigma^{\theta}, S P_{\omega}\right)= \begin{cases}2 & \text { if } \theta=\frac{r}{n} \omega \text { or } \frac{n-r}{n} \omega \\ 0 & \text { otherwise. }\end{cases}
$$

Proof. Note that

$$
\begin{aligned}
\rho \cdot \operatorname{Re} W_{r} & =\cos \frac{2 \pi r}{n} \operatorname{Re} W_{r}+\sin \frac{2 \pi r}{n} \operatorname{Im} W_{r}, \\
\rho \cdot \operatorname{Im} W_{r} & =-\sin \frac{2 \pi r}{n} \operatorname{Re} W_{r}+\cos \frac{2 \pi r}{n} \operatorname{Im} W_{r} \\
\cos ^{\omega}(t+\theta) & =\cos \left(\frac{2 \pi}{\omega} t\right) \cos \left(\frac{2 \pi}{\omega} \theta\right)-\sin \left(\frac{2 \pi}{\omega} t\right) \sin \left(\frac{2 \pi}{\omega} \theta\right),
\end{aligned}
$$




$$
\sin ^{\omega}(t+\theta)=\sin \left(\frac{2 \pi}{\omega} t\right) \cos \left(\frac{2 \pi}{\omega} \theta\right)+\cos \left(\frac{2 \pi}{\omega} t\right) \sin \left(\frac{2 \pi}{\omega} \theta\right) .
$$

Consequently,

$$
\begin{aligned}
& \rho \cdot\left(x_{1} \varepsilon_{1}+x_{2} \varepsilon_{2}+x_{3} \varepsilon_{3}+x_{4} \varepsilon_{4}\right) \\
& =x_{1} \cos ^{\omega}\left[\cos \frac{2 \pi r}{n} \operatorname{Re} W_{r}+\sin \frac{2 \pi r}{n} \operatorname{Im} W_{r}\right] \\
& -x_{1} \sin ^{\omega}\left[-\sin \frac{2 \pi r}{n} \operatorname{Re} W_{r}+\cos \frac{2 \pi r}{n} \operatorname{Im} W_{r}\right] \\
& +x_{2} \sin ^{\omega}\left[\cos \frac{2 \pi r}{n} \operatorname{Re} W_{r}+\sin \frac{2 \pi r}{n} \operatorname{Im} W_{r}\right] \\
& +x_{2} \cos ^{\omega}\left[-\sin \frac{2 \pi r}{n} \operatorname{Re} W_{r}+\cos \frac{2 \pi r}{n} \operatorname{Im} W_{r}\right] \\
& +x_{3} \cos ^{\omega}\left[\cos \frac{2 \pi r}{n} \operatorname{Re} W_{r}+\sin \frac{2 \pi r}{n} \operatorname{Im} W_{r}\right] \\
& +x_{3} \sin ^{\omega}\left[-\sin \frac{2 \pi r}{n} \operatorname{Re} W_{r}+\cos \frac{2 \pi r}{n} \operatorname{Im} W_{r}\right] \\
& +x_{4} \sin ^{\omega}\left[\cos \frac{2 \pi r}{n} \operatorname{Re} W_{r}+\sin \frac{2 \pi r}{n} \operatorname{Im} W_{r}\right] \\
& -x_{4} \cos ^{\omega}\left[-\sin \frac{2 \pi r}{n} \operatorname{Re} W_{r}+\cos \frac{2 \pi r}{n} \operatorname{Im} W_{r}\right] \\
& =x_{1} \cos \frac{2 \pi r}{n}\left[\cos ^{\omega} \operatorname{Re} W_{r}-\sin ^{\omega} \operatorname{Im} W_{r}\right] \\
& +x_{1} \sin \frac{2 \pi r}{n}\left[\cos ^{\omega} \operatorname{Im} W_{r}+\sin ^{\omega} \operatorname{Re} W_{r}\right] \\
& +x_{2} \cos \frac{2 \pi r}{n}\left[\sin ^{\omega} \operatorname{Re} W_{r}+\cos ^{\omega} \operatorname{Im} W_{r}\right] \\
& +x_{2} \sin \frac{2 \pi r}{n}\left[\sin ^{\omega} \operatorname{Im} W_{r}-\cos ^{\omega} \operatorname{Re} W_{r}\right] \\
& +x_{3} \cos \frac{2 \pi r}{n}\left[\cos ^{\omega} \operatorname{Re} W_{r}+\sin ^{\omega} \operatorname{Im} W_{r}\right] \\
& +x_{3} \sin \frac{2 \pi r}{n}\left[\cos ^{\omega} \operatorname{Im} W_{r}-\sin ^{\omega} \operatorname{Re} W_{r}\right] \\
& +x_{4} \cos \frac{2 \pi r}{n}\left[\sin ^{\omega} \operatorname{Re} W_{r}-\cos ^{\omega} \operatorname{Im} W_{r}\right] \\
& +x_{4} \cos \frac{2 \pi r}{n}\left[\sin ^{\omega} \operatorname{Im} W_{r}+\cos ^{\omega} \operatorname{Re} W_{r}\right] \\
& =\left(x_{1} \cos \frac{2 \pi r}{n}-x_{2} \sin \frac{2 \pi r}{n}\right) \varepsilon_{1}+\left(x_{1} \sin \frac{2 \pi r}{n}+x_{2} \cos \frac{2 \pi r}{n}\right) \varepsilon_{2} \\
& +\left(x_{3} \cos \frac{2 \pi r}{n}+x_{4} \sin \frac{2 \pi r}{n}\right) \varepsilon_{3}+\left(-x_{3} \sin \frac{2 \pi r}{n}+x_{4} \cos \frac{2 \pi r}{n}\right) \varepsilon_{4},
\end{aligned}
$$


and

$$
\begin{aligned}
& \left(x_{1} \varepsilon_{1}+x_{2} \varepsilon_{2}+x_{3} \varepsilon_{3}+x_{4} \varepsilon_{4}\right)(\cdot+\theta) \\
& =x_{1}\left[\cos ^{\omega} \cos \frac{2 \pi}{\omega} \theta-\sin ^{\omega} \sin \frac{2 \pi}{\omega} \theta\right] \operatorname{Re} W_{r} \\
& -x_{1}\left[\sin ^{\omega} \cos \frac{2 \pi}{\omega} \theta+\cos ^{\omega} \sin \frac{2 \pi}{\omega} \theta\right] \operatorname{Im} W_{r} \\
& +x_{2}\left[\sin ^{\omega} \cos \frac{2 \pi}{\omega} \theta+\cos ^{\omega} \sin \frac{2 \pi}{\omega} \theta\right] \operatorname{Re} W_{r} \\
& +x_{2}\left[\cos ^{\omega} \cos \frac{2 \pi}{\omega} \theta-\sin ^{\omega} \sin \frac{2 \pi}{\omega} \theta\right] \operatorname{Im} W_{r} \\
& +x_{3}\left[\cos ^{\omega} \cos \frac{2 \pi}{\omega} \theta-\sin ^{\omega} \sin \frac{2 \pi}{\omega}\right] \operatorname{Re} W_{r} \\
& +x_{3}\left[\sin ^{\omega} \cos \frac{2 \pi}{\omega} \theta+\cos ^{\omega} \sin \frac{2 \pi}{\omega} \theta\right] \operatorname{Im} W_{r} \\
& +x_{4}\left[\sin ^{\omega} \cos \frac{2 \pi}{\omega} \theta+\cos ^{\omega} \sin \frac{2 \pi}{\omega} \theta\right] \operatorname{Re} W_{r} \\
& -x_{4}\left[\cos ^{\omega} \cos \frac{2 \pi}{\omega} \theta-\sin ^{\omega} \sin \frac{2 \pi}{\omega} \theta\right] \operatorname{Im} W_{r} \\
& =x_{1} \cos \frac{2 \pi}{\omega} \theta\left[\cos ^{\omega} \operatorname{Re} W_{r}-\sin ^{\omega} \operatorname{Im} W_{r}\right] \\
& -x_{1} \sin \frac{2 \pi}{\omega} \theta\left[\sin ^{\omega} \operatorname{Re} W_{r}+\cos ^{\omega} \operatorname{Im} W_{r}\right] \\
& +x_{2} \cos \frac{2 \pi}{\omega} \theta\left[\sin ^{\omega} \operatorname{Re} W_{r}+\cos ^{\omega} \operatorname{Im} W_{r}\right] \\
& +x_{2} \sin \frac{2 \pi}{\omega} \theta\left[\cos ^{\omega} \operatorname{Re} W_{r}-\sin ^{\omega} \operatorname{Im} W_{r}\right] \\
& +x_{3} \cos \frac{2 \pi}{\omega} \theta\left[\cos ^{\omega} \operatorname{Re} W_{r}+\sin ^{\omega} \operatorname{Im} W_{r}\right] \\
& +x_{3} \sin \frac{2 \pi}{\omega} \theta\left[-\sin ^{\omega} \operatorname{Re} W_{r}+\cos ^{\omega} \operatorname{Im} W_{r}\right] \\
& +x_{4} \cos \frac{2 \pi}{\omega} \theta\left[\sin ^{\omega} \operatorname{Re} W_{r}-\cos ^{\omega} \operatorname{Im} W_{r}\right] \\
& +x_{4} \sin \frac{2 \pi}{\omega} \theta\left[\cos ^{\omega} \operatorname{Re} W_{r}+\sin ^{\omega} \operatorname{Im} W_{r}\right] \\
& =\left(x_{1} \cos \frac{2 \pi}{\omega} \theta+x_{2} \sin \frac{2 \pi}{\omega} \theta\right) \varepsilon_{1}+\left(-x_{1} \sin \frac{2 \pi}{\omega} \theta+x_{2} \cos \frac{2 \pi}{\omega} \theta\right) \varepsilon_{2} \\
& +\left(x_{3} \cos \frac{2 \pi}{\omega} \theta+x_{4} \sin \frac{2 \pi}{\omega}\right) \varepsilon_{3}+\left(-x_{3} \sin \frac{2 \pi}{\omega} \theta+x_{4} \cos \frac{2 \pi}{\omega} \theta\right) \varepsilon_{4} .
\end{aligned}
$$

Consequently, in order for the following equality

$$
\begin{aligned}
& \rho \cdot\left(x_{1} \varepsilon_{1}+x_{2} \varepsilon_{2}+x_{3} \varepsilon_{3}+x_{4} \varepsilon_{4}\right) \\
& \quad=\left(x_{1} \varepsilon_{1}+x_{2} \varepsilon_{2}+x_{3} \varepsilon_{3}+x_{4} \varepsilon_{4}\right)(\cdot+\theta)
\end{aligned}
$$


to hold, we must have

$$
\left\{\begin{array}{l}
x_{1} \cos \frac{2 \pi r}{n}-x_{2} \sin \frac{2 \pi r}{n}=x_{1} \cos \frac{2 \pi r}{n} \theta+x_{2} \sin \frac{2 \pi}{n} \theta \\
x_{1} \sin \frac{2 \pi r}{n}+x_{2} \cos \frac{2 \pi r}{n}=-x_{1} \sin \frac{2 \pi}{n} \theta+x_{2} \cos \frac{2 \pi}{\omega} \theta \\
x_{3} \cos \frac{2 \pi r}{n}+x_{4} \sin \frac{2 \pi r}{n}=x_{3} \cos \frac{2 \pi}{\omega} \theta+x_{4} \sin \frac{2 \pi}{\omega} \theta \\
x_{4} \cos \frac{2 \pi r}{n}-x_{3} \sin \frac{2 \pi r}{n}=x_{4} \cos \frac{2 \pi}{\omega} \theta-x_{3} \sin \frac{2 \pi}{\omega} \theta .
\end{array}\right.
$$

Consequently, (4.5) holds if and only if

$$
\begin{aligned}
& \theta=\frac{n-r}{n} \omega, \quad x_{3}=x_{4}=0, \quad x_{1}, x_{2} \in \mathbb{R}, \text { or } \\
& \theta=\frac{r}{n} \omega, \quad x_{1}=x_{2}=0, \quad x_{3}, x_{4} \in \mathbb{R}, \text { or } \\
& \theta \neq \frac{n-r}{n} \omega, \quad \frac{r}{n} \omega, \quad x_{1}=x_{2}=x_{3}=x_{4}=0 .
\end{aligned}
$$

Clearly,

$$
S P_{\omega}=\left\{x_{1} \varepsilon_{1}+x_{2} \varepsilon_{2}+x_{3} \varepsilon_{3}+x_{4} \varepsilon_{4} ; x_{1}, x_{2}, x_{3}, x_{4} \in \mathbb{R}\right\} .
$$

The conclusion thus follows.

Note that

$$
\Sigma_{m}=\{(\kappa, 1),(1,1)\}
$$

and

$$
\Sigma_{s}=\{(\kappa,-1),(1,1)\}
$$

are also subgroups of $\Gamma \times S^{1}$. A similar argument to that of Lemma 4.4 leads to the following:

Lemma 4.5. Assume that $\left|\alpha_{r} \beta\right|>1$ for some $r \in\left\{0, \ldots,\left[\frac{n}{2}\right]\right\}$, and $\alpha_{j} \neq \alpha_{r}$ for all $0 \leq j \neq r \leq\left[\frac{n}{2}\right]$. Then

$$
\begin{aligned}
\operatorname{Fix}\left(\Sigma_{m}, S P_{\omega}\right)=\left\{\sum_{i=1}^{4} x_{i} \varepsilon_{i} ; x_{3}\right. & =x_{1} \cos \frac{4 \pi}{n}-x_{2} \sin \frac{4 \pi}{n} \\
x_{4} & \left.=x_{1} \sin \frac{4 \pi}{n}+x_{2} \cos \frac{4 \pi}{n}, x_{1}, x_{2} \in \mathbb{R}\right\}
\end{aligned}
$$

and

$$
\begin{aligned}
\operatorname{Fix}\left(\Sigma_{s}, S P_{\omega}\right)=\left\{\sum_{i=1}^{4} x_{i} \varepsilon_{i} ; x_{3}\right. & =-x_{1} \cos \frac{4 \pi}{n}+x_{2} \sin \frac{4 \pi}{n}, \\
x_{4} & \left.=-x_{1} \sin \frac{4 \pi}{n}-x_{2} \cos \frac{4 \pi}{n}, x_{1}, x_{2} \in \mathbb{R}\right\} .
\end{aligned}
$$

In particular, $\operatorname{dim} \operatorname{Fix}\left(\Sigma_{m}, S P_{\omega}\right)=\operatorname{dim} \operatorname{Fix}\left(\Sigma_{s}, S P_{\omega}\right)=2$.

Lemmas 4.2-4.5 enable us to apply Theorem 2.1 to obtain the following result on the existence of smooth local Hopf bifurcations of wave solutions: 
Theorem 4.1. Assume that there exists $r \in\left\{0, \ldots,\left[\frac{n}{2}\right]\right\}$ such that $\left|\alpha_{r} \beta\right|>1$. Fix an integer $k$ such that $\tau_{r, k}>0$. Assume also that

(NR) $\sqrt{\left|\alpha_{i} \beta\right|^{2}-1} / \sqrt{\left|\alpha_{r} \beta\right|^{2}-1}$ is not an integer for any $i \in\left\{0, \ldots,\left[\frac{n}{2}\right]\right\}$ such that $i=r,\left|\alpha_{i} \beta\right|>1$ and $\tau_{i, k^{*}}=\tau_{r, k}$ for some integer $k^{*}$.

Then near $\tau_{r, k}$ there exist three branches of small-amplitude periodic solutions of (4.1) with period near $\frac{2 \pi}{\sqrt{\left|\alpha_{r} \beta\right|^{2}-1}}$ and satisfying

$$
\begin{gathered}
u_{i-1}(t)=u_{i}\left(t-\frac{r}{n} p\right), \quad i(\bmod n), \text { or } \\
u_{n-i}(t)=u_{i}(t), \quad i(\bmod n), \text { or } \\
u_{n-i}(t)=u_{i}\left(t-\frac{p}{2}\right), \quad i(\bmod n) .
\end{gathered}
$$

More precisely, we have

(i) there exist constants $a_{d}>0, \delta_{d}>0$ and continuously differential functions $\tau_{d}\left(x_{1}, x_{2}\right), p_{d}\left(x_{1}, x_{2}\right)$ and $p_{d}\left(x_{1}, x_{2}\right)$-periodic function $u_{d}\left(x_{1}, x_{2}\right)$, where $\left(x_{1}, x_{2}\right)^{T} \in \mathbb{R}^{2}$ and $\left|x_{1}\right|+\left|x_{2}\right|<a_{d}$, such that

(a) at $\tau=\tau_{d}\left(x_{1}, x_{2}\right), u_{d}\left(x_{1}, x_{2}\right)$ is a $p_{d}\left(x_{1}, x_{2}\right)$-periodic solution of (4.1) satisfying (4.6);

(b) $u_{d}(0,0)=0, p_{d}(0,0)=\frac{2 \pi}{\sqrt{\left|\alpha_{r} \beta\right|^{2}-1}}, \tau_{d}(0,0)=\tau_{r, k}$;

(c) $u_{d}\left(x_{1}, x_{2}\right)=x_{1} \varepsilon_{1}+x_{2} \varepsilon_{2}+o\left(\left|x_{1}\right|+\left|x_{2}\right|\right)$ as $\left|x_{1}\right|+\left|x_{2}\right| \rightarrow 0$.

Moreover, for $\left|\tau-\tau_{r, k}\right|<\delta,\left|p-\frac{2 \pi}{\sqrt{\left|\alpha_{r} \beta\right|^{2}-1}}\right|<\delta$, every $p$-periodic solution of (4.1) with $\sup |x(t)|_{t \in \mathbb{R}}<\delta$ and satisfying (4.6) must be of the above type.

(ii) there exist constants $a_{m}>0, \delta_{m}>0$ and continuously differential functions $\tau_{m}\left(x_{1}, x_{2}\right), p_{m}\left(x_{1}, x_{2}\right)$ and $p_{m}\left(x_{1}, x_{2}\right)$-periodic function $u_{m}\left(x_{1}, x_{2}\right)$, where $\left(x_{1}, x_{2}\right)^{T} \in \mathbb{R}^{2}$ and $\left|x_{1}\right|+\left|x_{2}\right|<a_{m}$, such that

(a) at $\tau=\tau_{m}\left(x_{1}, x_{2}\right), u_{m}\left(x_{1}, x_{2}\right)$ is a $p_{m}\left(x_{1}, x_{2}\right)$-periodic solution of (4.1) satisfying (4.7);

(b) $u_{m}(0,0)=0, p_{m}(0,0)=\frac{2 \pi}{\sqrt{\left|\alpha_{r} \beta\right|^{2}-1}}, \tau_{m}(0,0)=\tau_{r, k}$;

(c) $u_{m}\left(x_{1}, x_{2}\right)=\sum_{i=1}^{4} x_{i} \varepsilon_{i}+o\left(\left|x_{1}\right|+\left|x_{2}\right|\right)$ as $\left|x_{1}\right|+\left|x_{2}\right| \rightarrow 0$, where $x_{3}=$ $x_{1} \cos \frac{4 \pi}{n}-x_{2} \sin \frac{4 \pi}{n}$ and $x_{4}=x_{1} \sin \frac{4 \pi}{n}+x_{2} \cos \frac{4 \pi}{n}$;

Moreover, for $\left|\tau-\tau_{r, k}\right|<\delta_{m},\left|p-\frac{2 \pi}{\sqrt{\left|\alpha_{r} \beta\right|^{2}-1}}\right|<\delta_{m}$, every $p$-periodic solution of (4.1) with $\sup |x(t)|_{t \in \mathbb{R}}<\delta_{m}$ and satisfying (4.7) must be of the above type.

(iii) there exist constants $a_{s}>0, \delta_{s}>0$ and continuously differential functions $\tau_{s}\left(x_{1}, x_{2}\right), p_{s}\left(x_{1}, x_{2}\right)$ and $p_{s}\left(x_{1}, x_{2}\right)$-periodic function $u_{s}\left(x_{1}, x_{2}\right)$, where $\left(x_{1}, x_{2}\right)^{T} \in \mathbb{R}^{2}$ and $\left|x_{1}\right|+\left|x_{2}\right|<a_{s}$, such that

(a) at $\tau=\tau_{s}\left(x_{1}, x_{2}\right), u_{s}\left(x_{1}, x_{2}\right)$ is a $p_{s}\left(x_{1}, x_{2}\right)$-periodic solution of (4.1) satisfying (4.8);

(b) $u_{s}(0,0)=0, p_{s}(0,0)=\frac{2 \pi}{\sqrt{\left|\alpha_{r} \beta\right|^{2}-1}}, \tau_{s}(0,0)=\tau_{r, k}$;

(c) $u_{s}\left(x_{1}, x_{2}\right)=\sum_{i=1}^{4} x_{i} \varepsilon_{i}+o\left(\left|x_{1}\right|+\left|x_{2}\right|\right)$ as $\left|x_{1}\right|+\left|x_{2}\right| \rightarrow 0$, where $x_{3}=$ $-x_{1} \cos \frac{4 \pi}{n}+x_{2} \sin \frac{4 \pi}{n}$ and $x_{4}=-x_{1} \sin \frac{4 \pi}{n}-x_{2} \cos \frac{4 \pi}{n}$

Moreover, for $\left|\tau-\tau_{r, k}\right|<\delta_{s},\left|p-\frac{2 \pi}{\sqrt{\left|\alpha_{r} \beta\right|^{2}-1}}\right|<\delta_{s}$, every $p$-periodic solution of (4.1) with $\sup |x(t)|_{t \in \mathbb{R}}<\delta_{m}$ and satisfying (4.8) must be of the above type. 
Remark 4.1. Periodic solutions satisfying (4.6) are called discrete waves in the literature (cf. Fiedler [16] and Golubitsky, Stewart and Schaeffer [25] and references therein). They are also called synchronous oscillations (if $r \neq 0$ ) or phase-locked oscillations (if $r \neq 0$ ) as each neuron oscillates just like others except not necessarily in phase with each other. The existence of synchronous oscillation in ordinary/partial differential equations has been extensively studied (cf. Alexander and Auchmuty [3]) and some existence results have also been obtained for functional differential equations in conjunction with Turing rings with delayed coupling (see Krawcewicz and $\mathrm{Wu}[40]$ and $\mathrm{Wu}$ and Krawcewicz [55]. Periodic solutions satisfying (4.7) and (4.8) are called mirror-reflecting solutions and standing waves, respectively.

Remark 4.2. As will be shown in the next section, the non-resonance condition (NR) is not necessary for the existence of bifurcations of periodic solutions.

\section{Applications to Delayed neural networks: \\ Global CONTINUA OF DISCRETE WAVES}

Consider system (4.1) with a symmetric circulant interconnection matrix. Results in Section 4 show that, under certain conditions, system (4.1) has branches of periodic solutions $u(t)$ which satisfy the following property:

$$
u_{i-1}(t)=u_{i}\left(t-\frac{r}{n} p\right), \quad i(\bmod n),
$$

where $p$ is a period of $u(t)$ and $r$ is an integer satisfying $0 \leq r \leq n-1$.

Let $x(t)=u_{1}(t)$. Clearly, if $u(t)$ is a solution of (4.1) satisfying (5.1), the $x(t)$ is a solution of the following scalar functional differential equation

$$
\dot{x}(t)=-x(t)+\sum_{j=1}^{n} a_{j} f\left[x\left(t+\frac{(j-1) r}{n} p-\tau\right)\right] .
$$

Conversely, if $x(t)$ is a $p$-periodic solution of (5.2), then

$$
u(t)=\left(x(t), x\left(t+\frac{r}{n} p\right), \ldots, x\left(t+\frac{(n-1) r}{n} p\right)\right)^{T}
$$

is a $p$-periodic solution of (4.1) satisfying (5.1). Consequently, there exists a oneto-one correspondence between a $p$-periodic solution of the scalar equation (5.2) and a discrete wave satisfying (5.1) of the system (4.1).

When $r=0$, system (5.2) reduces to the following scalar delay-differential equation

$$
\dot{x}(t)=-x(t)+\left(\sum_{j=1}^{n} a_{j}\right) f(x(t-\tau)) .
$$

This equation has been extensively studied. In particular, Mallet-Paret and Nussbaum [45] proved the following:

Theorem 5.1. If $\beta \sum_{j=1}^{n} a_{j}<-1$, then for each

$$
\tau>\tau_{k}:=\frac{1}{\sqrt{\left|\sum_{j=1}^{n} a_{j} \beta\right|^{2}-1}}\left[\pi-\arccos \frac{1}{\left|\sum_{j-1}^{n} a_{j}\right|}+2 k \pi\right], \quad k=0,1, \ldots,
$$

equation (5.3) has at least $(k+1)$ periodic solutions $x^{1}(t), \ldots, x^{k+1}(t)$ such that the period of $x^{1}(t)$ is larger than $2 \tau$ and the period of $x^{m}(t), 1 \leq m \leq k$, is in the open 
interval $\left(\frac{\tau}{m+\frac{1}{2}}, \frac{\tau}{m}\right)$. Hence, for each $\tau>\tau_{k},(4.1)$ has $(k+1)$ distinct synchronous oscillations with periods in $(2 \tau, \infty)$ and $\left(\frac{\tau}{m+\frac{1}{2}}, \frac{\tau}{m}\right)$, respectively.

We now follow Mallet-Paret and Nussbaum [45] and call a periodic solution of (4.1) a slowly oscillatory solution if its period is larger than twice the delay, and a rapidly oscillatory solution if otherwise. So, Theorem 5.1 shows the coexistence of a slowly oscillatory synchronous oscillation and $k$ rapidly oscillatory synchronous oscillations for $\tau>\tau_{k}$ under the assumption $\beta \sum_{j=1}^{n} a_{j}<-1$.

In the case where $\sum_{j=1}^{n} a_{j}>0$, we have the following analog of Theorem 4.1.

Theorem 5.2. If $\beta \sum_{j=1}^{n} a_{j}>1$, then for each

$$
\tau>\tau_{k}:=\frac{1}{\sqrt{\left(\beta \sum_{j=1}^{n} a_{j}\right)^{2}-1}}\left[2 \pi-\arccos \frac{1}{\beta \sum_{j=1}^{n} a_{j}}+2 k \pi\right], \quad k=0,1, \ldots,
$$

equation (5.3) has $k+1$ periodic solutions $x^{1}(t), \ldots, x^{k+1}(t)$ with periods in $(\tau, 2 \tau)$, $\left(\frac{\tau}{m+1}, \frac{\tau}{m}\right), 1 \leq m \leq k$, respectively. Hence, for each $\tau>\tau_{k}$, (4.1) has $k+1$ synchronous oscillation with periods in $(\tau, 2 \tau),\left(\frac{\tau}{m+1}, \frac{\tau}{m}\right), 1 \leq m \leq k$, respectively.

We defer the proof of this result to Remark 5.1.

Due to the topological nature of our global bifurcation theorem, we are unable to describe the stability of the obtained synchronous oscillation. In fact, the following result shows that in certain situations, the obtained synchronous oscillations are all unstable.

Theorem 5.3. If $0 \leq \sum_{j=1}^{n} a_{j}<\beta^{-1}$, then the trivial solution of (5.3) is a global attractor. Hence, (4.1) has no synchronous oscillations for all $\tau \geq 0$. If $\beta \sum_{j=1}^{n} a_{j}>1$, then equation (5.3) has three equilibria $y^{*}<0<z^{*}$ such that almost every solution of (5.3) is convergent to either $y^{*}$ or $z^{*}$. In particular, (4.1) has no stable synchronous oscillation for all $\tau \geq 0$.

Proof. The result is trivial if $\sum_{j=1}^{n} a_{j}=0$. If $\sum_{j=1}^{n} a_{j}>0$, then property (TF) implies that equation (5.3) has only one equilibrium 0 if $\beta \sum_{j=1}^{n} a_{j}<1$, and has three equilibria $y^{*}<0<z^{*}$ such that $f^{\prime}\left(y^{*}\right), f^{\prime}\left(z^{*}\right)<\frac{1}{\sum_{j=1} a_{j}}$ if $\beta \sum_{j=1}^{n} a_{j}>1$. The solution semiflow generated by equation (5.3) is eventually strongly monotone, and hence the stability of an equilibrium $x^{*}$ is determined by the associated ordinary differential equation $\dot{x}=-x+\sum_{j=1}^{n} a_{j} f^{\prime}\left(x^{*}\right) x$ by Theorem 3.1 of Smith [52]. Consequently, if $\beta \sum_{j=1}^{n} a_{j}<1$, then the unique equilibrium 0 is asymptotically stable, and if $\beta \sum_{j=1}^{n} a_{j}>1$, then the trivial solution is unstable and other equilibria $y^{*}$ and $z^{*}$ are asymptotically stable. The generic convergence to the asymptotically stable equilibria is a consequence of the general theory of monotone dynamical systems developed by Hirsch [32] and Smith [52]. This completes the proof.

Throughout the remainder of this section, we assume

$$
\beta \sum_{j=1}^{n} a_{j}<1 .
$$

It can be easily shown that under this assumption, 0 is the only equilibrium of (5.2) for all $p, \tau>0$. Our goal is to apply the global bifurcation theorem in Section 3 to investigate the maximal continuation of each branch of discrete waves obtained 
in Section 4. (The discussion of the maximal continua of standing waves and mirror-reflecting waves is similar and thus omitted). The existence results in the previous section is local in character; the amplitude is small and the delay $\tau$ is close to the bifurcation values $\tau_{r, k}$. We will show that the existence of discrete waves (synchronous/phase-locked oscillations) is preserved as $\tau$ moves away form these bifurcation values. Clearly, as $\tau$ moves away from $\tau_{r, k}$, the amplitude of discrete waves may increase. Consequently, we obtain large-amplitude periodic solutions.

First of all, we establish some a priori bounds for possible periodic solutions of (5.2).

Lemma 5.1. If $x(t)$ is a non-constant periodic solution of (5.2), then $|x(t)|<1$ for all $t \in \mathbb{R}$.

Proof. Let $t^{*} \in \mathbb{R}$ such that $\left|x\left(t^{*}\right)\right|=\sup |x(t)|_{t \in \mathbb{R}}$. Then $x\left(t^{*}\right) \neq 0$ and $\frac{d}{d t} x^{2}\left(t^{*}\right)$ $=0$. But $|f(x(t))|<1$ for all $t \in \mathbb{R}$ and

$$
\begin{aligned}
& \frac{d}{d t} x^{2}\left(t^{*}\right)=2 x\left(t^{*}\right)\left[-x\left(t^{*}\right)+\sum_{j=1}^{n} a_{j} f\left(x\left(t^{*}+\frac{(j-1) r}{n} p-\tau\right)\right)\right] \\
& <2\left[-x^{2}\left(t^{*}\right)+\left|x\left(t^{*}\right)\right| \sum_{j=1}^{n}\left|a_{j}\right|\right] \\
& =-2\left|x\left(t^{*}\right)\right|\left[\left|x\left(t^{*}\right)\right|-\sum_{j=1}^{n}\left|a_{j}\right|\right] \\
& =-2\left|x\left(t^{*}\right)\right|\left[\left|x\left(t^{*}\right)\right|-1\right] .
\end{aligned}
$$

Consequently, $\left|x\left(t^{*}\right)\right|<1$. This completes the proof.

Next, we exclude some periodic solutions with specific periods.

Lemma 5.2. If the diagonal elements of $J$ are zero, then system (4.1) has no non-constant periodic solutions of period $\tau$. Consequently, (5.2) with $p=\tau$ has no non-constant periodic solution of period $\tau$.

Proof. If $u(t)$ is a period solution of (4.1) of period $\tau$, then $u(t)$ solves the system of ordinary differential equations

$$
\dot{u}_{i}=-u_{i}+\sum_{j=1}^{n} J_{i j} f\left(u_{j}\right), \quad 1 \leq i \leq n .
$$

For this system of ordinary differential equations, Hopfield [33] showed that the time derivative of the energy function

$$
E=-\frac{1}{2} \sum_{i, j} J_{i j} f\left(u_{i}\right) f\left(u_{j}\right)+\sum_{i=1}^{n} \int_{0}^{f\left(u_{i}\right)} f^{-1}(v) d v
$$

is

$$
\frac{d E}{d t}=-\sum_{i=1}^{n} f^{\prime}\left(u_{i}\right)\left[u_{i}(t)-\sum_{j=1}^{n} J_{i j} f\left(u_{j}\right)\right]^{2} .
$$


Therefore, by the well-known invariance principle, each solution of (5.5) is convergent to an equilibrium. Consequently, (5.5) (and thus (4.1)) has no non-constant periodic solution of period $\tau$. This completes the proof.

Lemma 5.3. Assume that there exists a positive integer $s$ such that $\gamma_{s} f(y) / y<1$ for $y \neq 0$, where

$$
\gamma_{s}=\max \left\{\operatorname{Re} \sum_{j=1}^{n} a_{j} z^{(j-1) s-1} ; z^{n s}=1\right\},
$$

then equation (5.2) has no non-constant $r^{-1} n s \tau$-periodic solution (here, we assume $r \neq 0)$.

Proof. Suppose that $x(t)$ is an $r^{-1} n s \tau$-periodic solution of (5.2) with $p=r^{-1} n s \tau$. Then

$$
\dot{x}(t)=-x(t)+\sum_{j=1}^{n} a_{j} f[x(t+(j-1) s \tau-\tau)]
$$

Clearly, $x(t)$ is also $n s \tau$-periodic. Let

$$
\begin{aligned}
& y_{j}(t)=x(t+(j-1) \tau), \quad j(\bmod n s) \\
& y=\left(y_{1}, \ldots, y_{n s}\right)^{T}, \\
& A=\operatorname{circ}(\underbrace{0, \ldots, 0, a_{2}}_{s}, \underbrace{0, \ldots, 0, a_{3}}_{s}, \ldots, \underbrace{0, \ldots, 0, a_{n}}_{s}, \underbrace{0, \ldots, 0, a_{1}}_{s}) \\
& F(y)=\left(f\left(y_{1}\right), \ldots, f\left(y_{n s}\right)\right)^{T} .
\end{aligned}
$$

Then

$$
\dot{y}=-y+A F(y) .
$$

By Lemma 1.1 of Nussbaum [50] for the spectral analysis of circulant matrices, we have

$$
\begin{aligned}
& (-A z, z) \geq \min \left\{\operatorname{Re} \sum_{j=1}^{n}\left(-a_{j}\right) z^{(j-1) s-1} ; z^{n s}=1\right\} \sum_{i=1}^{n s} z_{i}^{2} \\
& =-\max \left\{\operatorname{Re} \sum_{j=1}^{n} a_{j} z^{(j-1) s-1} ; z^{n s}=1\right\} \sum_{i=1}^{n s} z_{i}^{2} \\
& =-\gamma_{s} \sum_{i=1}^{n s} z_{i}^{2}, z \in \mathbb{R}^{n s} .
\end{aligned}
$$

That is,

$$
(A z, z) \leq \gamma_{s} \sum_{i=1}^{n s} z_{i}^{2}
$$


Consequently, for $V(y)=\sum_{i=1}^{n} \int_{0}^{y_{i}} f(s) d s$, along solutions of (5.6), we have

$$
\begin{aligned}
\frac{d}{d t} V(y(t))=F(y)^{T}[-y+A F(y)] \\
\quad=\sum_{i=1}^{n s} y_{i} f\left(y_{i}\right)+(A F(y), F(y)) \\
\leq-\sum_{i=1}^{n s} f\left(y_{i}\right) y_{i}\left[1-\gamma_{s} \frac{f\left(y_{i}\right)}{y_{i}}\right] .
\end{aligned}
$$

Therefore, if $\gamma_{s} \frac{f(y)}{y}<1$ for all $y \neq 0$, then $\frac{d}{d t} V(y(t))<0$ along nontrivial solutions of (5.6). This implies that (5.6) has no non-constant periodic solutions. Hence, $x(t)$ must be constant. This completes the proof.

Theorem 5.4. Assume that

(i) (5.4) is satisfied;

(ii) there exists an integer $r \in\left\{0, \ldots,\left[\frac{n}{2}\right]\right\}$ such that $\left|\alpha_{r} \beta\right|>1$;

(iii) there exist an integer $k$ and two nonnegative numbers $q_{r, k, 1}<q_{r, k, 2}$ such that $\tau_{r, k}>0$,

$$
q_{r, k, 1}<\frac{2 \pi}{\theta_{r}-\arccos \frac{1}{\left|\alpha_{r} \beta\right|}+2 k \pi}<q_{r, k, 2}
$$

and (5.2) has non non-constant periodic solutions of period $q_{r, k, 1} \tau$ or $q_{r, k, 2} \tau$.

Then for every $\tau>\tau_{r, k}$, system (4.1) has a discrete wave (a p-periodic solution) satisfying $u_{i-1}(t)=u_{i}\left(t-\frac{r}{n} p\right)$ for $t \in \mathbb{R}, i(\bmod n)$ and $q_{r, k, 1}<p / \tau<q_{r, k, 2}$.

Proof. We regard $(\tau, p)$ as parameters and apply Theorem 3.3. By (5.4), we can easily show that $(0, \tau, p)$ is the only stationary solution of $(5.2)$ and the corresponding characteristic matrix

$$
\Delta_{(0, \tau, p)}(\lambda)=\lambda+1-\sum_{j=1}^{n} a_{j} \beta e^{\lambda\left(\frac{j-1}{n} r p-\tau\right)}
$$

is clearly continuous in $(\tau, p, \lambda) \in \mathbb{R}_{+} \times \mathbb{R}_{+} \times \mathbb{C}$. This justifies (A1)-(A3) of Theorem 3.3 for the considered equation $(5.2)$.

To locate centers, we consider

$$
\begin{aligned}
\Delta_{(0, \tau, p)} & \left(i \frac{2 m \pi}{p}\right)=i \frac{2 m \pi}{p}+1-\sum_{j=1}^{n} a_{j} \beta \xi^{(j-1) r m} e^{-i r \frac{2 m \pi}{p}} \\
= & i \frac{2 m \pi}{p}+1-\alpha_{m r} \beta e^{-i \tau \frac{2 m \pi}{p}} \\
= & q\left(i \frac{2 m \pi}{p}\right)
\end{aligned}
$$

where

$$
q(\lambda)=\lambda+1-a_{m r} \beta e^{-\tau \lambda}
$$

Using results in Lemma 4.1 and notations in Lemma 4.2, we can show that for each fixed $r,(0, \tau, p)$ is a center if and only if there exist an integer $m \geq 1$ and an integer 
$k$ such that

$$
\left\{\begin{array}{l}
\left|\alpha_{m r} \beta\right|>1 \\
p=\frac{2 m \pi}{\sqrt{\left|\alpha_{m r} \beta\right|^{2}-1}} \\
\tau=\tau_{m r, k}>0
\end{array}\right.
$$

In particular, $\left(0, \tau_{r, k}, \frac{2 \pi}{\sqrt{\left|\alpha_{r} \beta\right|^{2}-1}}\right)$ is a center and all centers are isolated. In fact, the set of centers is countable and can be expressed as

$$
\Delta=\left\{\left(0, \tau_{m r, k}, \frac{2 m \pi}{\sqrt{\left|\alpha_{m r} \beta\right|^{2}-1}}\right) ;\left|\alpha_{m r} \beta\right|>1, \tau_{m r, k}>0\right\} .
$$

We now fix $r \in\{0, \ldots, n-1\}$ such that $\left|\alpha_{r} \beta\right|>1$. Then $\left(0, \tau_{r, k}, \frac{2 \pi}{\sqrt{\left|\alpha_{r} \beta\right|^{2}-1}}\right)$ is an isolated center and $1 \in J\left(0, \tau_{r}^{*}, \frac{2 \pi}{\sqrt{\left|\alpha_{r} \beta\right|^{2}-1}}\right)$.

Consider $q(\lambda)$ with $m=1$. By Lemma 4.1 there exist $\varepsilon>0, \delta>0$ and a smooth curve $\lambda:\left(\tau_{r, k}-\delta, \tau_{r, k}+\delta\right) \rightarrow \mathbb{C}$ such that $q(\lambda(\tau))=0,\left|\lambda(\tau)-i \frac{2 \pi}{\sqrt{\left|\alpha_{r} \beta\right|^{2}-1}}\right|<\varepsilon$ for all $\tau \in\left[\tau_{r, k}-\delta, \tau_{r, k}+\delta\right]$, and

$$
\lambda\left(\tau_{r, k}\right)=i \frac{2 \pi}{\sqrt{\left|\alpha_{r} \beta\right|^{2}-1}},\left.\frac{d}{d \tau} \operatorname{Re} \lambda(\tau)\right|_{\tau=\tau_{r, k}}>0 .
$$

Let

$$
\Omega_{\varepsilon}=\left\{(0, p) ; 0<u<\varepsilon,\left|p-\frac{2 \pi}{\sqrt{\left|\alpha_{r} \beta\right|^{2}-1}}\right|<\varepsilon\right\} .
$$

Clearly, if $\left|\tau-\tau_{r, k}\right| \leq \delta$ and $(u, p) \in \partial \Omega_{\varepsilon}$ such that $\operatorname{det} \Delta_{(0, \tau, p)}\left(u+i \frac{2 \pi}{p}\right)=0$, then $\tau=\tau_{r, k}, u=0$ and $p=\frac{2 \pi}{\sqrt{\left|\alpha_{r} \beta\right|^{2}-1}}$. This justifies (A4) for $m=1$. Moreover, if we put

$$
H_{m}^{ \pm}\left(0, \tau_{r, k}, \frac{2 \pi}{\sqrt{\left|\alpha_{r} \beta\right|^{2}-1}}\right)(u, p)=\Delta_{\left(0, \tau_{r, k} \pm \delta, p\right)}\left(u+i m \frac{2 \pi}{p}\right),
$$

then at $m=1$, we have

$$
\begin{aligned}
\gamma_{m}\left(0, \tau_{r, k}, \frac{2 \pi}{\sqrt{\left|\alpha_{r} \beta\right|^{2}-1}}\right) \\
=\operatorname{deg}_{B}\left(H_{m}^{-}\left(0, \tau_{r, k}, \frac{2 \pi}{\sqrt{\left|\alpha_{r} \beta\right|^{2}-1}}\right), \Omega_{\varepsilon}\right) \\
\quad-\operatorname{deg}_{B}\left(H_{m}^{+}\left(0, \tau_{r, k}, \frac{2 \pi}{\sqrt{\left|\alpha_{r} \beta\right|^{2}-1}}\right), \Omega_{\varepsilon}\right) \\
=-1 .
\end{aligned}
$$

By Theorem 3.3, we conclude that the connected component $\mathcal{C}\left(0, \tau_{r, k}, \frac{2 \pi}{\sqrt{\left|\alpha_{r} \beta\right|^{2}-1}}\right)$ through $\left(0, \tau_{r, k}, \frac{2 \pi}{\sqrt{\left|\alpha_{r} \beta\right|^{2}-1}}\right)$ in $\Sigma_{r}$ is nonempty, where

$$
\Sigma_{r}=\operatorname{cl}\{(x, \tau, p) ; x \text { is a } p \text {-periodic solution of }(5.2)\} .
$$


Using the same argument as above, we can show that the first crossing number of each center is always -1 . Therefore, we can use (3.2) to exclude alternative (ii) of Theorem 3.3. That is, we can conclude that $\mathcal{C}\left(0, \tau_{r, k}, \frac{2 \pi}{\sqrt{\left|\alpha_{r} \beta\right|^{2}-1}}\right)$ is unbounded.

Lemma 5.1 implies that the projection of $\mathcal{C}\left(0, \tau_{r, k}, \frac{2 \pi}{\sqrt{\left|\alpha_{r} \beta\right|^{2}-1}}\right)$ onto the $x$-space is bounded. Also, note that the proof of Lemma 5.2 implies that the system (4.1) with $\tau=0$ has no non-constant periodic solution. Therefore, the projection of $\mathcal{C}\left(0, \tau_{r, k}, \frac{2 \pi}{\sqrt{\left|\alpha_{r} \beta\right|^{2}-1}}\right)$ onto the $\tau$-space is bounded below. Assumption (iii) implies that, if $(x, \tau, p) \in \mathcal{C}\left(0, \tau_{r, k}, \frac{2 \pi}{\sqrt{\left|\alpha_{r} \beta\right|^{2}-1}}\right)$, then $\tau q_{r, k, 1}<p<\tau q_{r, q, 2}$. This shows that in order for $\mathcal{C}\left(0, \tau_{r, k}, \frac{2 \pi}{\sqrt{\left|\alpha_{r} \beta\right|^{2}-1}}\right)$ to be unbounded, the projection of $\mathcal{C}\left(0, \tau_{r, k}, \frac{2 \pi}{\sqrt{\left|\alpha_{r} \beta\right|^{2}-1}}\right)$ onto $\tau$-space must be unbounded. Consequently, the projection of $\mathcal{C}\left(0, \tau_{r, k}, \frac{2 \pi}{\sqrt{\left|\alpha_{r} \beta\right|^{2}-1}}\right)$ onto $\tau$-space must be an interval $[\alpha, \infty)$ with $0<\alpha<\tau_{r, k}$. This shows that for each $\tau>\tau_{r, k},(5.2)$ has a non-constant periodic solution with a period in $\left(\tau q_{r, k, 1}, \tau q_{r, k, 2}\right)$. This completes the proof.

Remark 5.1. We can now give a proof of Theorem 5.2. In fact, Chow and MalletParet [8] have proved that equation (5.3) has no non-constant periodic solution of period $2 \tau$. Consequently, it has no non-constant periodic solution of period $\frac{\tau}{m}$ or $\frac{2 \tau}{m+1}=\frac{\tau}{m+\frac{1}{2}}$ for all positive integers $m$. Therefore, Theorem 5.2 becomes a special case of Theorem 5.4 where $r=0, \alpha_{r}=\sum_{j=1}^{n} a_{j} ; \theta_{r}=0, \tau_{r, k}=\tau_{k-1}$, $q_{r, k+1,1}=\frac{1}{k+1}, q_{r, k+1,2}=\frac{1}{k}$ for all $k=1,2, \ldots$, and $q_{r, 1,1}=1, q_{r, 1,2}=2$.

Remark 5.2. We can always take $q_{1, k, 1}=0$ if we do not need good estimation of the lower bound of the period of periodic solutions. Moreover, we can use Lemma 5.2 and Lemma 5.3 to determine $q_{r, k, 1}$ and $q_{r, k, 2}$ in some concrete neural networks, see the next section for details.

Remark 5.3. Applying Theorem 3.2 and employing the same argument as that for Theorem 5.4, we can obtain a local bifurcation theorem for (4.1): If there exist $r \in$ $\left\{0, \ldots,\left[\frac{n}{2}\right]\right\}$ and an integer $k$ such that $\left|\alpha_{r} \beta\right|>1$ and $\tau_{r, k}>0$, then near $\tau_{r, k},(4.1)$ has a branch of small-amplitude $p$-periodic solution satisfying $u_{i-1}(t)=u_{i}\left(t-\frac{1}{n} p\right)$, $i(\bmod n), t \in R$. Note that we do not require non-resonance condition, but we cannot describe the smoothness, the isotropy group and the asymptotic form of the bifurcated wave solution.

\section{EXAMPLES AND FURTHER DISCUSSIONS}

In this section, we concentrate on some specific networks to discuss some implications and limitations of our general results. For simplicity, we will only consider synchronous/phase-locked oscillations and omit the discussion about standing waves and mirror-reflecting waves.

6.A. All-Excitatory Networks. For this special network, as $f$ is an increasing function and the interconnection matrix has positive off diagonal elements, the solution semiflow is eventually strongly monotone and hence the convergence to equilibria is the dominant dynamics. More precisely, we have

Theorem 6.1. Consider equation (4.1) with the interconnection matrix $J_{E}$. 
(i) If $\beta=f^{\prime}(0)<1$, then for any $\tau>0$ the unique equilibrium $(0, \ldots, 0)$ is a global attractor. That is, any solution of $(4.1)$ converges to $(0, \ldots, 0)$ as $t \rightarrow \infty$.

(ii) If $\beta=f^{\prime}(0)>1$, then equation (4.1) has three and only three equilibria $\left(y^{*}, \ldots, y^{*}\right),(0, \ldots, 0)$ and $\left(z^{*}, \ldots, z^{*}\right)$ with $y^{*}<0<z^{*}, f\left(y^{*}\right)=y^{*}$, $f\left(z^{*}\right)=z^{*} .(0, \ldots, 0)$ is unstable, $\left(y^{*}, \ldots, y^{*}\right)$ and $\left(z^{*}, \ldots, z^{*}\right)$ are asymptotically stable and almost every solution of (4.1) is convergent to either $\left(y^{*}, \ldots, y^{*}\right)$ or $\left(z^{*}, \ldots, z^{*}\right)$ as $t \rightarrow \infty$. Moreover, there exists a solution $u(t)$ of (4.1) such that $u_{i}(t)<u_{i}(s)$ if $t<s, 1 \leq i \leq n$, and $\lim _{t \rightarrow-\infty} u_{i}(t)=0$, $\lim _{t \rightarrow \infty} u_{i}(t)=z^{*}$; and there exists a solution $v(t)$ of (4.1) such that $v_{i}(t)<v_{i}(s)$ if $t>s, 1 \leq i \leq n$, and $\lim _{t \rightarrow-\infty} v_{i}(t)=0, \lim _{t \rightarrow \infty} v_{i}(t)=y^{*}$.

Proof. Let $\left(x_{1}, \ldots, x_{n}\right)$ be an equilibrium of (4.1). Then

$$
x_{i}=\frac{1}{n-1} \sum_{j \neq i} f\left(x_{j}\right), \quad 1 \leq i \leq n .
$$

Hence,

$$
x_{i}-x_{j}=\frac{1}{n-1}\left[f\left(x_{j}\right)-f\left(x_{i}\right)\right]
$$

and from the increasing property of $f$ it follows that $x_{i}=x_{j}$ for all $1 \leq i, j \leq n$. Therefore, $\left(x_{1}, \ldots, x_{n}\right)$ is an equilibrium of (4.1) if and only if $x=f(x)$ and $x_{i}=x$ for all $1 \leq i \leq n$. Consequently, using property (TF) we conclude that (4.1) has a unique equilibrium $(0, \ldots, 0)$ if $\beta<1$, and has three equilibria $\left(y^{*}, \ldots, y^{*}\right)$, $(0, \ldots, 0),\left(z^{*}, \ldots, z^{*}\right)$ when $f\left(y^{*}\right)=y^{*}<0<z^{*}=f\left(z^{*}\right)$ if $\beta>1$. In the latter case, we also have $f^{\prime}\left(y^{*}\right), f^{\prime}\left(z^{*}\right)<1$.

We now consider the case where $\beta<1$. As $f$ is strictly increasing and $J_{E}$ has positive off diagonal elements, the general theory of Smith [52] shows that the solution semiflow generated by (4.1) is eventually strongly monotone and the zero solution $(0, \ldots, 0)$ of $(4.1)$ is asymptotically stable if and only if the zero solution of the associated ordinary differential equation

$$
\dot{x}_{i}=-x_{i}+\sum_{j \neq i} \frac{\beta}{n-1} f\left(x_{j}\right)
$$

is asymptotically stable. It can be easily shown that eigenvalues of (6.1) at the trivial solution are $-1+\beta \alpha_{r}, 0 \leq r \leq n-1$, where

$$
\alpha_{r}= \begin{cases}1 & \text { if } r=0 \\ -\frac{1}{n-1} & \text { if } 1 \leq r \leq n-1\end{cases}
$$

Consequently, $\beta<1$ implies that the zero solution of (6.1) is asymptotically stable. As each solution of $(4.1)$ is clearly bounded, $(0, \ldots, 0)$ is the only equilibrium and is asymptotically stable. By results in Smith [52], the zero solution is a global attractor.

In the case where $\beta>1$, using the same argument as above we can verify that the zero solution $(0, \ldots, 0)$ is unstable, and $\left(y^{*}, \ldots, y^{*}\right),\left(z^{*}, \ldots, z^{*}\right)$ are asymptotically stable. Again, by results in Hirsch [32] and Smith [52], almost every solution of (4.1) is convergent to either $\left(y^{*}, \ldots, y^{*}\right)$ or $\left(z^{*}, \ldots, z^{*}\right)$, and there are monotonically heteroclinic orbits connecting $(0, \ldots, 0)$ to $\left(y^{*}, \ldots, y^{*}\right)$ and to $\left(z^{*}, \ldots, z^{*}\right)$. This completes the proof. 
Remark 6.1. The above result shows that the generic dynamics of (4.1) is the convergence to equilibria. This is independent of the length of the delay $\tau$. The only interesting phenomena is the bifurcation of the pitchfork type: as the neuron gain moves through value 1 , the zero solution becomes unstable, and two asymptotic stable equilibria appear in either side of the origin in the ferromagnetic direction $\left(u_{i}=1\right.$ for all $\left.1 \leq i \leq n\right)$. This phenomena was observed in Marcus and Westervelt [47].

Let us now try to apply Theorem 5.4 and Remark 5.3 to discuss the occurrence of discrete waves. Clearly,

$$
\begin{aligned}
\alpha_{r} & =\frac{1}{n-1}\left[\xi^{r}+\xi^{2 r}+\cdots+\xi^{(n-1) r}\right] \\
& = \begin{cases}1 & \text { if } r=0 \\
-\frac{1}{n-1} & \text { if } 1 \leq r \leq n-1\end{cases}
\end{aligned}
$$

Let

$$
\begin{aligned}
\tau_{0, k} & =\frac{1}{\sqrt{\beta^{2}-1}}\left[2 k \pi-\arccos \frac{1}{\beta}\right] \text { if } \beta>1 \text { and } k \geq 1 ; \\
\tau_{k} & =\frac{n-1}{\sqrt{\beta^{2}-(n-1)^{2}}}\left[\pi-\arccos \frac{n-1}{\beta}+2 k \pi\right] \text { if } \beta>n-1 \text { and } k \geq 0 .
\end{aligned}
$$

Applying Remark 5.3, we get

Theorem 6.2. Consider equation (4.1) with the interconnection matrix $J_{E}$.

(i) If $\beta>1$, then near $\tau_{0, k}, k \geq 1$, there exists a branch of small-amplitude synchronous oscillations, i.e. a branch of small-amplitude periodic solution with period near $2 \pi / \sqrt{\beta^{2}-1}$ and satisfying $u_{i}(t)=u_{1}(t)$ for $1 \leq i \leq n$ and $\tau \in \mathbb{R}$

(ii) If $\beta>n-1$, then near $\tau_{k}, k \geq 0$, there exist $(n-1)$ branches of smallamplitude phase-locked oscillations with distinct phase differences, i.e. $(n-$ 1) branches of small-amplitude phase-locked oscillations with period near $2 \pi(n-1) / \sqrt{\beta^{2}-(n-1)^{2}}$ and satisfying $u_{i-1}(t)=u_{i}\left(t-\frac{r}{n} p\right)$ for $t \in \mathbb{R}$, $i(\bmod n), 1 \leq r \leq n-1$.

Remark 6.2. By Theorem 6.1, any synchronous/phase-locked oscillation obtained above must be unstable. Consequently, in all-excitatory networks, delay may cause discrete waves but these waves can hardly be observed in experiments. An interesting phenomena here is the simultaneous occurrence of $(n-1)$ branches of phase-locked oscillations with distinct phase differences. We should also mention that if $(n-1) \sqrt{\frac{\beta^{2}-1}{\beta^{2}-(n-1)^{2}}}$ is not a positive integer, then we can apply Theorem 2.1 to discuss the smoothness and directions of these $(n-1)$ branches of phase-locked oscillations.

6.B. Rings of Inhibitory Neurons. We next consider symmetrically connected inhibitory rings of neurons, where

$$
\begin{aligned}
\alpha_{r} & =-\frac{1}{2}\left[\xi^{r}+\xi^{(n-1) r}\right] \\
& = \begin{cases}-1 & \text { if } r=0, \\
-\cos \frac{2 \pi r}{n} & \text { if } r=1, \ldots, n-1 .\end{cases}
\end{aligned}
$$


Consequently, $\alpha_{i}=\alpha_{j}$ if and only if $i+j=n$.

Note that

$$
\begin{aligned}
\gamma_{s} & =\max \left\{\operatorname{Re}\left(-\frac{1}{2} z^{s-1}-\frac{1}{2} z^{(n-1) s-1}\right) ; z^{n s}=1\right\} \\
& =\max \left\{-\frac{1}{2} \operatorname{Re}\left(z^{s-1}+z^{-s-1}\right) ; z^{n s}=1\right\} \\
& =\max \left\{-\frac{1}{2}\left[\cos \frac{2 \pi j(s-1)}{n s}+\cos \frac{2 \pi j(s+1)}{n s}\right] ; 0 \leq j \leq n s-1\right\} \\
& =\max \left\{-\cos \frac{2 \pi j}{n s} \cos \frac{2 \pi j}{n} ; 0 \leq j \leq n s-1\right\} .
\end{aligned}
$$

In particular,

$$
\gamma_{1}=\max \left\{-\left(\cos \frac{2 \pi j}{n}\right)^{2} ; 0 \leq j \leq n-1\right\} \leq 0 .
$$

Therefore,

$$
\gamma_{1} f(y) / y \leq 0<1 \quad \text { for all } y \neq 0
$$

Let

$$
\tau_{0, k}=\frac{1}{\sqrt{\beta^{2}-1}}\left[\pi-\arccos \frac{1}{\beta}+2 k \pi\right], \quad \text { if } \beta>1, k \geq 0,
$$

and

$$
\tau_{r, k}=\frac{1}{\sqrt{\left|\beta \cos \frac{2 \pi r}{n}\right|^{2}-1}}\left\{\begin{array}{l}
{\left[2(k+1) \pi-\arccos \frac{1}{\left|\beta \cos \frac{2 \pi r}{n}\right|}\right]} \\
\text { if } \beta \cos \frac{2 \pi r}{n}<-1, k \geq 0, \\
{\left[\begin{array}{r}
\frac{1}{\left|\beta \cos \frac{2 \pi r}{n}\right|}+2 k \pi \\
\text { if } \beta \cos \frac{2 \pi r}{n}>1, k \geq 0 .
\end{array}\right.}
\end{array}\right.
$$

Theorem 6.3. Consider system (4.1) with the interconnection matrix $J_{I E}$.

(i) If $\beta>1$, then for each $\tau>\tau_{0, k}$, (4.1) has $(k+1)$ distinct synchronous oscillations with periods in $(2 \tau, \infty)$ and $\left(\frac{\tau}{m+\frac{1}{2}}, \frac{\tau}{m}\right), 1 \leq m \leq k$, respectively;

(ii) If $\frac{r}{n} \in\left(\frac{1}{4}, \frac{3}{4}\right)$ and $\beta \cos \frac{2 \pi \tau}{n}<-1$, then for each $\tau>\tau_{r k, k}$, (4.1) has $(k+1)$ phase-locked oscillations with periods in $\left(\tau, \frac{n}{r} \tau\right)$ and $\left(\frac{\tau}{m+1}, \frac{\tau}{m}\right), 1 \leq m \leq k$, respectively and satisfying $u_{i-1}(t)=u_{i}\left(t-\frac{r}{n} p\right), i(\bmod n), t \in \mathbb{R}$;

(iii) If $\frac{r}{n} \in\left(0, \frac{1}{4}\right) \cup\left(\frac{3}{4}, 1\right)$ and $\beta \cos \frac{2 \pi r}{n}>1$, then for each $\tau>\tau_{r, k}$, (4.1) has $(k+1)$ phase-locked oscillations with periods in $\left(2 \tau, \frac{n}{r} \tau\right)\left(\right.$ if $\left.\frac{r}{n} \in\left(0, \frac{1}{4}\right)\right)$ or $\left(2 \tau, \frac{n}{n-r} \tau\right)$ if $\left.\frac{r}{n} \in\left(\frac{3}{4}, 1\right)\right)$, and $\left(\frac{\tau}{m+1}, \frac{\tau}{m}\right), 1 \leq m \leq k$, respectively and satisfying $u_{i-1}(t)=u_{i}\left(t-\frac{r}{n} p\right), i(\bmod n), t \in \mathbb{R}$.

Proof. (i) is an immediate consequence of Theorem 5.1, where $\sum_{j=1}^{n} a_{j}=-1<0$.

(ii) Assume $\frac{r}{n} \in\left(\frac{1}{4}, \frac{3}{4}\right)$ and $\beta \cos \frac{2 \pi r}{n}<-1$. Note that

$$
\frac{1}{k+1}=\frac{2 \pi}{2(k+1) \pi}<\frac{2 \pi}{2(k+1) \pi-\arccos \frac{1}{\left|\beta \cos \frac{2 \pi r}{n}\right|}}<\frac{2 \pi}{2(k+1) \pi-\frac{\pi}{2}}=\frac{1}{k+\frac{3}{4}} \text {. }
$$


So,

$$
\begin{aligned}
& 1<\frac{2 \pi}{2(k+1) \pi-\arccos \frac{1}{\left|\beta \cos \frac{2 \pi r}{n}\right|}}<\frac{4}{3}<\frac{n}{r}, \quad \text { if } k=0, \\
& \frac{1}{k+1}<\frac{2 \pi}{2(k+1) \pi-\arccos \frac{1}{\left|\beta \cos \frac{2 \pi r}{n}\right|}}<\frac{1}{k}, \quad \text { if } k \geq 1 .
\end{aligned}
$$

Since $\gamma_{1} \leq 0$, by Lemma 5.3, equation (5.2) has no non-constant $\frac{n}{r} \tau$-periodic solution. Moreover, by Lemma 5.2, equation (5.2) has no non-constant $\frac{\tau}{m}$-periodic solution for any positive integer $m$. Therefore, we can apply Theorem 5.4 to obtain the required result, where

$$
q_{r, 0,1}=1, \quad q_{r, 0,2}=\frac{n}{r}, \quad q_{r, k, 1}=\frac{1}{k+1}, \quad q_{r, k, 2}=\frac{1}{k}, \quad k \geq 1 .
$$

This proves (ii), and (iii) can be justified similarly.

Remark 6.3. Note that $\cos \frac{2 \pi r}{n}=\cos \frac{2 \pi(n-r)}{n}$. So, $\tau_{r, k}=\tau_{n-r, k}$ for all $r \in\{1, \ldots$, $n-1\}$ and $k \geq 0$. Therefore, if $r \neq \frac{n}{2}$, then at each $\tau_{r, k}$ there are two branches of phase-locked oscillations which are conjugate in the sense that if solutions along one branch satisfy $u_{i-1}(t)=u_{i}\left(t-\frac{r}{n} p\right)$, then solutions along another branch satisfy $u_{i-1}(t)=u_{i}\left(t+\frac{r}{n} p\right)\left(\right.$ as $\left.u_{i}\left(t-\frac{n-r}{n} p\right)=u_{i}\left(t+\frac{r}{n} p\right)\right)$. This implies that Theorem 5.3 actually guarantees the existence of $2(k+1)$ phase-locked oscillations in either case (ii) or case (iii).

Remark 6.4. If $n=4 q+m$ for some nonnegative integer $q$ and integer $m \in$ $\{0,1,2,3\}$, then $\frac{r}{n} \in\left(0, \frac{1}{4}\right) \cup\left(\frac{3}{4}, 1\right)$ if and only if

$$
r \in\left\{\begin{array}{l}
\{1,2, \ldots, q, n-1, \ldots, n-q) \quad \text { if } m \neq 0, \\
\varnothing \quad \text { if } m=0 \text { and } q \leq 1, \\
\{1, \ldots, q-1, n-1, \ldots, n-q+1\} \quad \text { if } m=0 \text { and } q \geq 2 .
\end{array}\right.
$$

Consequently, if

$$
\beta>\max _{\frac{r}{n} \in\left(0, \frac{1}{4}\right) \cup\left(\frac{3}{4}, 1\right)} \frac{1}{\cos \frac{2 \pi r}{n}},
$$

then for sufficiently large $\tau$ satisfying

$$
\tau>\left\{\begin{array}{l}
\max \left\{\tau_{0,0}, \tau_{1,0}, \ldots, \tau_{q, 0}\right\} \quad \text { if } m \neq 0, \\
\max \left\{\tau_{0,0}, \tau_{1,0}, \ldots, \tau_{q-1,0}\right\} \quad \text { if } q \geq 2, m=0, \\
\tau_{0,0} \quad \text { if } m=0, q \leq 1,
\end{array}\right.
$$

system (4.1) has at least $\left(2 q^{*}+1\right)$ slowly oscillatory periodic solutions, one of which is synchronous oscillation and others are phase-locked oscillations, where

$$
q^{*}= \begin{cases}q & \text { if } m \neq 0, \\ q-1 & \text { if } m=0, q \geq 2, \\ 0 & \text { if } m-0,0 \leq q \leq 1 .\end{cases}
$$

6.C. All-inhibitory networks and rings of excitatory neurons. Using the same argument as that in 6 .A and 6.B, we can show that all-inhibitory networks exhibit the coexistence of synchronous and phase-locked oscillations for large delay and neural gains. It is also possible for a ring of excitatory neurons to possess 
synchronous/phase-locked oscillations, but these oscillations are all unstable and the dominant dynamics is the convergence to equilibria.

\section{ACKnowledgement}

This research was supported by Natural Sciences and Engineering Research Council of Canada and by Faculty of Arts Research Grants of York University. Part of the results was reported in various meetings including Workshop on Geometric Methods in Ordinary Differential Equations, Oberwolfach, March of 1995; Geometric Dynamics and Its Applications, Berkeley, October of 1993; The 2nd International Conference on Differential Equations, Athens, August of 1993; SIAM Conference on Applications of Dynamical Systems, Snowbird, October of 1992. The author has benefited very much from discussions with and encouragement from J. Bélair, B. Fiedler, A. Herz, M. Hirsch, J. Mallet-Paret, J. Mawhin, K. Mischaikow, R. Nussbaum, K. Schmidt, P. van den Driessche and H. O. Walther.

\section{REFERENCES}

[1] J. C. Alexander, Bifurcation of zeros of parametrized functions, J. Funct. Anal. 29 (1978), 37-53. MR 80j:58023

[2] J. C. Alexander and G. Auchmuty, Global branching of waves, Manuscripta Math. 27 (1979), 159-166. MR 80g:58021

[3] _ Global bifurcations of phase-locked oscillators, Arch. Rational Mech. Anal. 93 (1986), 253-270. MR 87j:34090

[4] J. C. Alexander and P. M. Fitzpatrick, The homotopy of certain spaces of nonlinear operators, and its relation to global bifurcation of the fixed points of parametrized condensing operators, J. Funct. Anal. 34 (1979), 87-106. MR 81k:58023

[5] J. C. Alexander and J. Yorke, Global bifurcation of periodic orbits, Amer. J. Math. 100 (1978), 263-292. MR 57:14046

[6] J. Bélair, Stability in a model of a delayed neural network, J. Dynamics and Differential Equations 5 (1993), 607-623. MR 94i:34150

[7] R. D. Braddock and P. van den Driessche, On the stability of differential-difference equations, J. Austral. Math. Soc. 19(B) (1976), 358-370. MR 56:6058

[8] S. N. Chow and J. Mallet-Paret, The fuller index and global Hopf bifurcation, J. Differential Equations 29 (1978), 66-85. MR 58:11665

[9] S. N. Chow, J. Mallet-Paret and J. A. Yorke, Global Hopf bifurcation from a multiple eigenvalue, Nonlinear Anal. 2 (1978), 753-763. MR 80a:58015

[10] M. A. Cohn and S. Grossberg, Absolute stability of global pattern formation and parallel memory storage by competitive neural networks, IEEE Trans. Systems Man Cybernet. 13 (1983), 815-826. MR 85e:92002

[11] E. N. Dancer, Boundary-value problems for ordinary differential equations on infinite intervals, Proc. London Math. Soc. 30 (1975), 76-94. MR 52:867

[12] G. Dylawerski, K. Gęba, J. Jodel and W. Marzantowicz, $S^{1}$-equivalent degree and the Fuller index, Ann. Polon. Math. 52 (1991), 243-280. MR 92g:58105

[13] L. H. Erge, K. Gęba, W. Krawcewicz, and J. Wu, $S^{1}$-degree and global Hopf bifurcation theory of functional differential equations, J. Differential Equations 98 (1992), 277-298. MR 93f: 58034

[14] C. Fenske, Analytische Theorie des Abbildungrades für Abbildungen in Banachraumes, Math. Nachr. 48 (1971), 279-290. MR 49:7857

[15] B. Fiedler, An index for global Hopf bifurcation in parabolic systems, J. Reine Angew. Math. 359 (1985), 1-36. MR 87c:58024

[16] _ Global Bifurcation of Periodic Solutions with Symmetry, Lecture Notes in Math., Vol. 1309, Springer-Verlag, New York, 1988. MR 90c:58125

[17] P. M. Fitzpatrick, Homotopy, linearization, and bifurcation, Nonlinear Anal. 12 (1988), 171184. MR 89g:58039

[18] A. Frumkin and E. Moses, Physicality of the Little model, Physical Review (A), 34 (1986), $714-716$. 
[19] F. B. Fuller, An index of fixed point type for periodic orbits, Amer. J. Math. 89 (1967), 133-148. MR 35:497

[20] K. Geba, W. Krawcewicz and J. Wu, An equivariant degree with applications to symmetric bifurcation problems, I: construction of the degree, Proc. London Math. Soc. 69 (1994), 377398. MR 95g:58025

[21] K. Geba an W.. Marzantowicz, Global bifurcation of periodic solutions, Topological Methods in Nonlinear Analysis 1 (1993), 67-93. MR 94e:58024

[22] E. Goles-Chacc, F. Fogelman-Soulie and D. Pellegrin, Decreasing energy functions as a tool for studying threshold networks, Discrete Appl. Math. 12 (1985), 261-277. MR 87d:68084

[23] E. Goles and G. Y. Vichniac, Lyapunov functions for parallel neural networks, in "Neural Networks for Computing (J. S. Denber ed.)", Amer. Inst. Phy., New York (1986), pp. 165-181.

[24] M. Golubitsky and I. N. Stewart, Hopf bifurcation in the presence of symmetry, Arch. Rational Mech. Anal. 87 (1985), 107-165. MR 86g:58034

[25] M. Golubitsky, I. Stewart and D. G. Schaeffer, Singularities and Groups in Bifurcation Theory, Vol. 2, Springer-Verlag, New York, 1988. MR 89m:58038

[26] G. Grinstein, C. Jayaprakash and P. He, Statistical mechanics of probabilistic automata, Phys. Rev. Lett. 55 (1985), 2527-2530. MR 87c:82029

[27] J. Hale, Theory of Functional Differential Equations, Springer-Verlag, New York 1977. MR 58:22904

[28] _ Nonlinear Oscillations in Equations with Delays, in "Nonlinear Oscillations in Biology", pp. 157-185, Lectures in Applied Mathematics, Vol. 17, Amer. Math. Soc., Providence, RI, 1979. MR 81d:34062

[29] D. Hassard, N. D. Kazarinoff and Y. Wan, Theory and Applications of Hopf Bifurcation, Cambridge Univ. Press, Cambridge, 1981. MR 82j:58089

[30] A. Herz, B. Salzer, R. Kühn and J. L. van Hemmen, Hebbian learning reconsidered: representation of static and dynamic objects in associative neural nets, Biol. Cybern. 60 (1989), 457-467.

[31] G. Hetzer and V. Stallbohm, Global behaviour of bifurcation branches and the essential spectrum, Math. Nachr. 86 (1978), 343-360. MR 80g:47076

[32] M. W. Hirsch, Stability and convergence in strongly monotone dynamical systems, J. Reine Angew. Math. 383 (1988), 1-53. MR 89c:58108

[33] J. J. Hopfield, Neural networks and physical systems with emergent collective computational abilities, Proc. Nat. Acad. Sci. 79 (1982), 2554-2558. MR 83g:92024

[34] J. J. Hopfield, Neurons with graded response have collective computational properties like those of two-stage neurons, Proc. Nat. Acad. Sci. 81 (1984), 3088-3092.

[35] J. Ize, Bifurcation theory for Fredholm operators, Mem. Amer. Math. Soc. 17 (1976). MR 54:13649

[36] _ Obstruction theory and multiparameter Hopf bifurcation, Trans. Amer. Math. Soc. 209 (1985), 757-792.

[37] J. Ize, I. Massabó and A. Vignoli, Degree theory for equivariant maps, (I). Trans. Amer. Math. Soc. 315 (1989), 433-510. MR 90a:58030

[38] _ Degree theory for equivariant maps, (II). The general $S^{1}$-action, Mem. Amer. Math. 100 (1992). MR 93d:58030

[39] W. Krawcewicz, P. Vivi and J. Wu, Computation formulae of an equivalent degree with applications to symmetric bifurcations, Nonlinear Studies 4 (1997), 89-120. CMP 97:14

[40] W. Krawcewicz and J. Wu, Theory and applications of Hopf bifurcations in symmetric functional differential equations, Nonlinear Analysis, in press.

[41] W. Krawcewicz, H. Xia and J. Wu, Global bifurcation theory for condensing fields and neutral equations with applications to lossless transmission problems, Canadian Appl. Math. Quarterly 1 (1993), 167-220. MR 94g:47077

[42] B. W. Levinger, A folk theorem in functional differential equations, J. Differential Equations 4 (1980), 612-619. MR 37:6566

[43] W. A. Little, Existence of persistent states in the brain, Math. Biosci. 19 (1974), 101-120.

[44] W. A. Little and G. L. Shaw, Analytic study of the memory storage capacity of a neural network, Math. Biosci. 39 (1978), 281-290.

[45] J. Mallet-Paret and R. D. Nussbaum, Global continuation and asymptotic behaviour for periodic solutions of a differential-delay equation, Ann. Mate. Pura. Appl. 145 (1986), 33128. MR 88e:34127 
[46] J. Mallet-Paret and J. Yorke, Snakes: oriented families of periodic orbits, their sources, sinks and continuation, J. Differential Equations 43 (1992), 419-450. MR 84a:58071

[47] C. M. Marcus and R. M. Westervelt, Stability of analog neural networks with delay, Physical Review A 39 (1989), 347-359. MR 89m:92025

[48] R. D. Nussbaum, A global bifurcation theorem with applications to functional differential equations, J. Funct. Anal. 19 (1975), 319-338. MR 52:6516

[49] _ A Hopf global bifurcation theorem for retarded functional differential equations, Trans. Amer. Math. Soc. 238 (1978), 139-164. MR 81h:34082

[50] _ Circulant matrices and differential-delay equations, J. Differential Equations 60 (1985), 201-217. MR 87d:34112

[51] P. H. Rabinowitz, Some global results for nonlinear eigenvalue problems, J. Functional Anal. 7 (1971), 487-513. MR 46:745

[52] H. L. Smith, Monotone semiflows generated by functional differential equations, J. Differential Equations 66 (1987), 420-442. MR 88j:34155

[53] C. A. Stuart, Some bifurcation theory for $k$-set contractions, Proc. London Math. Soc. 27 (1973), 531-550. MR 48:12178

[54] A. Vanderbauwhede, Local Bifurcation and Symmetry, Res. Notes Math., 75, Pitman, Boston, 1982. MR 85f: 58026

[55] J. Wu and W. Krawcewicz, Discrete waves and phase-locked oscillations in the growth of a single-species population over a patchy environment, Open Systems and Information Dynamics 1 (1992), 127-147.

Department of Mathematics and Statistics, York University, North York, Ontario, CANADA M3J 1P3

E-mail address: wujh@mathstat.yorku.ca 